A dimensão de representação de álgebras de Artin Antônio Marcos Correa Neri

DISSERTAÇÃO APRESENTADA

$\mathrm{AO}$

INSTITUTO DE MATEMÁTICA E ESTATÍSTICA

DA

UNIVERSIDADE DE SÃO PAULO

PARA

OBTENÇÃO DO GRAU DE MESTRE

EM

CIÊNCIAS

Área de Concentração: Álgebra

Orientador: Prof. Dr. Flávio Ulhoa Coelho

Durante a elaboração deste trabalho

o autor recebeu apoio financeiro da FAPESP.

São Paulo, maio de 2006 


\section{A dimensão de representação \\ de álgebras de Artin}

Este exemplar corresponde à redação final da dissertação devidamente corrigida e defendida por Antônio Marcos Correa Neri e aprovada pela comissão julgadora.

São Paulo, 5 de maio de 2006.

Banca examinadora:

- Prof. Dr. Flávio Ulhoa Coelho (orientador) - IME-USP

- Prof. Dr. Alegria Gladys Chalom de Oliveira - IME-USP

- Prof. Dr. Edson Ribeiro Álvares - UFPR 
Àquela que, companheira em tudo, é motivo e razão. 


\section{Agradecimentos}

Algumas pessoas merecem agradecimentos especiais de minha parte por conta desta dissertação. Antes delas, porém, devo agradecer a Deus Pai, por ter-me contemplado com Sua Graça, aquilo que inadvertidamente as pessoas insistem em chamar de sorte.

Merece agradecimento meu orientador, Professor Flávio, por ter sido extremamente paciente e solícito sempre; por ter me oferecido oportunidade concreta para que eu concluísse este mestrado, ensinando, auxiliando, incentivando; por ter sido exemplo de pessoa.

Merecem agradecimentos todos os amigos que estiveram envolvidos direta ou indiretamente com esta empreitada, nominalmente: Zeca e Rapha, pela bela maneira acolhedora de ser, pelas conversas e risadas; Péterson e Marcone, que, junto com o Zeca, me deram abrigo, e posteriormente, morada; mais que isso, junto com Ricardo Damm ofereceram momentos gostosos de oração; Eliza e Márcia, presentes em cada momento desse mestrado, que puxavam a orelha quando necessário, que faziam festa sempre, sendo irmãs como alguém nunca terá; ao Márcio e Clayton, que foram companheiros nos últimos dias em São Paulo; o pessoal do PET e o Fedor e também os professores, que fizeram crescer o desejo desse mestrado; toda minha família de irmãos em Campo Largo, grupo de jovens chamado Água Viva, que lutou comigo durante todo meu trabalho desde a faculdade e por quem não me abandona a saudade todo o tempo.

Merecem agradecimentos meus pais, minha irmã e meu irmão, pessoas que deixei quando fui para São Paulo, de quem senti muita falta sempre e em cada momento; pelo apoio todo dia.

Merece agradecimento, este sim, mais que especial, aquela que, companheira em tudo, é motivo e razão de ser desse trabalho, a quem devo o desejo do começo e a felicidade do fim; aquela a quem pesou a distância, a quem alegrou cada reencontro, e mesmo longe, aceitou o desafio de viver uma vida inteira comigo, minha amada esposa Janete. 


\section{Resumo}

O conceito de dimensão de representação foi definido no início da década de 70 por Maurice Auslander com a intenção de medir o tipo de representação de uma álgebra de Artin $\Lambda$. Este número, $\inf \left\{\operatorname{gl} \cdot \operatorname{dim}\left(\operatorname{End}_{\wedge}(M)^{\text {op }}\right)\right\}$, onde $M$ é gerador-cogerador de $\bmod (\Lambda)$, caracteriza as álgebras de tipo finito, ou seja, $\Lambda$ é de tipo finito se, e somente se, rep. $\operatorname{dim}(\Lambda) \leqslant 2$. Por longo tempo não foi possível apresentar resultados sobre esta dimensão, mas com o avanço das técnicas na Teoria de Representações, muitos autores recentemente têm mostrado novos caminhos para entender a dimensão de representação. Entre esses resultados, destacam-se a finitude da dimensão de representação, a relação com a conjectura finitística e as definições equivalentes de dimensão de representação que permitem calculá-la para algumas classes de álgebra. Esta dissertação apresenta estas definições equivalentes e exemplos de seu uso para calcular a dimensão de representação de álgebras de tipo finito, álgebras hereditárias de tipo infinito, álgebras coladas à direita e álgebras coladas à esquerda. São ainda comentados alguns resultados que aparecem na literatura a respeito da dimensão de representação. 


\section{Abstract}

The concept of representation dimension was introduced in the beginning of 70's by Maurice Auslander with the intention to measure the representation type of an Artin algebra $\Lambda$. This number, $\inf \left\{g l \cdot \operatorname{dim}\left(\operatorname{End}_{\wedge}(M)^{o p}\right)\right\}$, where $M$ is a generator-cogenerator of $\bmod (\Lambda)$, characterizes representation finite algebras, or either, $\Lambda$ is of representation finite type if, and only if, $\operatorname{rep} \operatorname{dim}(\Lambda) \leqslant 2$. For long time it was not possible to present results about representation dimension, but with the advance of techniques in the Representation Theory, many authors recently have shown new way to reach the understanding of representation dimension. Among these results, it could be distinguished finiteness of representation dimension, the relation with finitistic conjecture and the equivalent definitions for representation dimension that allow to calculate it for some classes of algebras. This work presents these equivalent definitions and examples of their use to calculate the representation dimension of finite representation algebras, hereditary algebras of infinite type, right glued and left glued algebras. Some results that appear in literature regarding the representation dimension are commented in the last section. 


\section{Sumário}

Introdução

1 Álgebras e Módulos 3

1.1 Algumas equivalências . . . . . . . . . . . . . . . . 9

1.2 Funtores Coerentes . . . . . . . . . . . . . . . . . . . . 11

2 A dimensão de representação $\quad 18$

2.1 A motivação . . . . . . . . . . . . . . . . . . . . . . . . 18

2.2 A definição . . . . . . . . . . . . . . . . . . . . . . . . . . . . 22

2.3 Uma outra caracterização . . . . . . . . . . . . . . . . . . . . . . 32

3 Alguns Exemplos 38

3.1 Dimensão de Representação 3 . . . . . . . . . . . . . . . . . . . . . . 40

3.1.1 A dimensão de representação de álgebras hereditárias . . . . . . . . 40

3.1 .2 Álgebras coladas e Álgebras Laura. . . . . . . . . . . . . . . . . . 43

3.2 Outros resultados . . . . . . . . . . . . . . . . . . . 48 


\section{Introdução}

No começo da década de 70 do século passado, Maurice Auslander mostrou em "Representation Dimension of Artin Algebras" ([Aus71]) que a classe de álgebras de Artin de tipo finito está fortemente ligada à classe de álgebras de Artin com dimensão global no máximo 2 e dimensão dominante no mínimo 2. Para ser mais exato, considerando Morita-equivalência, essas classes estão em correspondência biunívoca.

Isso o motivou a examinar com mais cuidado o que significava para uma álgebra ter dimensão dominante no mínimo 2, e dessa forma surgiu a "dimensão de representação", como sendo um meio de "medir" quão longe uma álgebra de Artin está de ser de tipo de representação finito.

Por muito tempo não houve nenhum avanço significativo sobre o assunto, já que ficaram muitas perguntas sem resposta. Entretanto, recentemente o tema ganhou renovado interesse, pois com o desenvolvimento técnico da teoria foi possível alargar a classe de álgebras para as quais se pode conhecer melhor a dimensão de representação. Isso pode parecer pouco, mas um resultado de K. Igusa e G. Todorov [IT02] relacionou a dimensão de representação com a conjectura finitística, que está ligada a outras conjecturas relevantes. Aquele artigo mostra que, para álgebras com rep. $\operatorname{dim}(\Lambda) \leq 3$, a conjectura finitística é válida.

É sabido que a dimensão de representação é sempre finita e por muito tempo pensou-se que ela não passasse de 3, mas recentemente R. Rouquier anunciou que conseguiu construir uma álgebra com dimensão de representação igual a 4, e comentou que o exemplo poderia ser estendido para todos os naturais maiores que 4.

Este trabalho objetiva colocar o máximo de informação possível a respeito da dimensão de representação, apresentando essas relações e algumas classes de álgebras para as quais já se sabe que ela é no máximo 3. 
No primeiro capítulo, serão apresentados conceitos, definições e resultados relevantes para o desenvolvimento dos capítulos posteriores. Entre esses resultados, o de mais destaque é a equivalência entre duas categorias, apresentado no Lema 1.23.

As definições básicas sobre a dimensão de representação são apresentadas no segundo capítulo, onde se pode observar a motivação e a definição dada por Auslander, uma caracterização importante que ele mesmo apresentou em termos de geradores e cogeradores, e uma caracterização devida a Coelho e Platzeck, em termos de resoluções de aproximações. Destacam-se os Teoremas 2.11 e 2.21 .

No último capítulo, são apresentados alguns exemplos que exploram as técnicas desenvolvidas no segundo capítulo, calculando a dimensão de representação de álgebras de Artin de tipo finito, álgebras hereditárias e álgebras coladas.

Também aparecem no último capítulo, um resumo dos resultados que aparecem na literatura, apresentando limitantes para a dimensão de representação de algumas classes de álgebras, como as Álgebras Laura, álgebras que são mergulhos em radicais de álgebras de tipo finito, entre outros resultados. 


\section{Capítulo 1}

\section{Álgebras e Módulos}

As álgebras e módulos constituem um campo de estudo da matemática bastante desenvolvido. Muitos trabalhos foram apresentados em forma de livros, artigos, apresentações em encontros científicos. Os resultados básicos usados nessa dissertação podem ser encontrados em textos como os livros de J.J. Rotman [Rot79], de F. Anderson e K. Fuller [AF92] e de I. Assem [Ass97].

Este trabalho baseia-se em um tipo específico de álgebras (as álgebras de Artin) e módulos sobre estas álgebras. Nesta seção, são apresentadas as definições e resultados gerais, que podem ser encontrados na literatura publicada sobre o assunto. As notações seguem de perto as usadas por Auslander em [Aus71], e por Auslander, Reiten e Smal $\varnothing$, no texto clássico sobre teoria de representações de álgebras de Artin, [ARS95].

O uso de técnicas e conceitos categóricos e homológicos é inevitável. As definições de categorias, funtores e aplicações naturais são consideradas conhecidas, e qualquer uso que se faça destas palavras pode ser facilmente reconhecido quando comparado a algumas das referências já citadas ([Aus71, ARS95, Ass97]).

A menos de menção em contrário, ao longo do texto a palavra módulo significará módulo à esquerda e anel significará anel com unidade.

Seja R um anel. Denota-se por $\operatorname{Mod}(R)$ a categoria dos R-módulos à esquerda e por $\bmod (R)$ a subcategoria plena de $\operatorname{Mod}(R)$ formada pelos R-módulos finitamente gerados.

Um R-módulo $M$ é indecomponivel quando não pode ser escrito de forma alguma como soma direta de seus submódulos.

Se o fato de que um módulo $M$ é isomorfo a $N$ é denotado por $M \cong N$, então a 
relação binária " $M$ se relaciona com $N \Leftrightarrow M \cong N$ " define em $\bmod (R)$ uma relação de equivalência. Cada classe de equivalência definida por essa relação chama-se isoclasse. A notação ind( $R$ ) será usada para representar a subcategoria plena de $\bmod (R)$ cujos objetos são um representante de cada isoclasse $[M]$, com $M$ indecomponível. Um anel $R$ é um anel com tipo de representação finito quando, a menos de isomorfismo, há apenas um número finito de módulos indecomponíveis.

O teorema abaixo, conhecido com Teorema de Krull-Schmidt, é bastante usado ao longo do texto.

1.1 Teorema. [AF92, 12.9] Considere $\mathrm{R}$ um anel artiniano e seja $\mathrm{M}$ um R-módulo de comprimento finito. Então $M$ admite uma decomposição $\mathrm{M}=\mathrm{N}_{1} \oplus \mathrm{N}_{2} \oplus \ldots \oplus \mathrm{N}_{\mathrm{r}}$, com $\mathrm{N}_{\mathrm{i}} \in \operatorname{ind}(\mathrm{R})$, para todo $i \in\{1, \ldots, \mathrm{r}\}$. Essa decomposição é única a menos de isomorfismo, ou seja, se $\mathrm{M}=\mathrm{N}^{\prime}{ }_{1} \oplus \mathrm{N}^{\prime}{ }_{2} \oplus \ldots \oplus \mathrm{N}^{\prime}{ }_{\mathrm{s}}$, então $\mathrm{r}=\mathrm{s}$ e para cada $\mathrm{i} \in\{1, \ldots, \mathrm{r}\}$, existe um $j \in\{1, \ldots, s\}$, tal que $\mathbf{N}_{\mathbf{i}} \cong \mathbf{N}^{\prime}{ }_{j}$.

Lembre que $M$ tem comprimento finito se e só se $M$ é módulo artiniano e noetheriano [AF92, 11.1].

Então, se R é um anel artiniano, todo R-módulo finitamente gerado $M$ é de comprimento finito e portanto, pode ser decomposto de forma única em soma direta de seus submódulos indecomponíveis.

Este é o caso das álgebras de Artin. Estas são estruturas algébricas que generalizam as bem estudadas álgebras de dimensão finita sobre corpos (veja por exemplo [DK94] ou [CLS82]).

1.2 Definição. Seja R um anel.

- Um R-módulo $\Lambda$ é uma R-álgebra quando $\wedge$ tem estrutura de anel e vale, para cada $u, v \in \Lambda$ e para cada $r \in R$

$$
(r u) v=r(u v)=u(r v)
$$

- Uma R-álgebra $\Lambda$ é uma R-álgebra de Artin quando R é um anel comutativo artiniano e ${ }_{\mathrm{R}} \wedge$ é um R-módulo finitamente gerado. 
1.3 Exemplo. Seja k um corpo. Então, toda k-álgebra de dimensão finita $V$ é uma álgebra de Artin, já que k é um anel artiniano e $V$, sendo de dimensão finita, é finitamente gerada.

Denota-se por $\mathrm{R}^{\mathrm{op}}$ ao anel oposto de $\mathrm{R}$, ou seja, ao anel cujo grupo aditivo é idêntico ao de $\mathrm{R}$, mas com operação de multiplicação $*$ definida por $a * b=b a$, onde ba é o produto de $b$ por $a$ em $R$.

Se $\Lambda$ é uma R-álgebra, o anel $\Lambda^{o p}$ também é R-módulo, e portanto faz sentido perguntar se $\Lambda^{\text {op }}$ é uma $R$-álgebra. Para mostrar que isso é verdadeiro, basta observar que vale, para cada par $a, b \in \Lambda^{\text {op }}$ e para cada $r \in R$ :

$$
\begin{aligned}
r(a * b) & =r(b a)=(r b) a=a * r b \\
& =r(b a)=b(r a)=r a * b
\end{aligned}
$$

Então, fica bem definida, para uma dada R-álgebra $\Lambda$, a sua álgebra oposta, que será denotada por $\Lambda^{\text {op }}$.

As álgebras de Artin preservam propriedades importantes das álgebras sobre corpos. Os resultados abaixo podem ser observados no capítulo II de [ARS95]. Lembre que $\operatorname{Hom}_{\Lambda}(M, N)$ denota o grupo abeliano dos morfismos do módulo $M$ para o módulo $N$, e que $\operatorname{End}_{\Lambda}(M)$ denota o anel dos endomorfismos de $M$.

1.4 Proposição. Seja $\wedge$ uma R-álgebra de Artin.

- Se $\mathrm{Z}(\Lambda)$ indica o centro do anel $\Lambda$, então $\Lambda$ é uma $\mathrm{Z}(\Lambda)$-álgebra de Artin. Assim, $\mathrm{R}$ será omitido daqui em diante.

- $\wedge$ é um anel artiniano.

- Se $M \in \bmod (\Lambda)$, então $\operatorname{End}_{\Lambda}(M)$ é uma álgebra de Artin.

- Se $\wedge \wedge=n_{1} P_{1} \oplus n_{2} P_{2} \oplus \ldots \oplus n_{r} P_{r}$ é a decomposição de $\wedge$ segundo o teorema de Krull-Schmidt, então os $\Lambda$-módulos projetivos finitamente gerados indecomponíveis estão no conjunto $\left\{\mathrm{P}_{\mathfrak{i}}\right\}_{\mathfrak{i}=1}^{r}$. 
1.5 Definição. Seja $M \in \bmod (\Lambda)$, com $\Lambda$ uma álgebra de Artin. A subcategoria plena de $\bmod (\Lambda)$ formada por somandos diretos de somas finitas de cópias de $M$, chamada de subcategoria gerada por $M$, será denotada por add $(M)$. Assim, um $\Lambda$-módulo $N$ está em $\operatorname{add}(M)$ quando existem $E \in \bmod (\Lambda)$ e $n \in \mathbb{N}$ tais que $N \oplus E=n M$.

Não é difícil ver que a caracterização abaixo é verdadeira.

1.6 Proposição. Seja $\mathrm{M} \in \bmod (\Lambda)$. Então $\mathrm{N} \in \operatorname{add}(\mathrm{M})$ se e somente se $\mathrm{N}$ é isomorfo a uma soma direta de somandos indecomponíveis de $\mathrm{M}$.

Observe que add $(M)$ é uma subcategoria fechada para somas e somandos diretos e isomorfismos.

As definições abaixo são dadas sobre a categoria de módulos sobre uma álgebra de Artin $\Lambda$, mas algumas delas podem ser dadas em um contexto mais geral.

Um epimorfismo $f: M \rightarrow N$ de $\bmod (\Lambda)$ é dito epimorfismo essencial quando satisfaz, para qualquer $\mathrm{g}: \mathrm{L} \rightarrow \mathrm{M}$, a condição abaixo:

$$
f g \text { é epimorfismo } \Rightarrow g \text { é epimorfismo. }
$$

Dualmente, um monomorfismo $\mathrm{g}: \mathrm{L} \rightarrow \mathrm{M}$ em $\bmod (\Lambda)$ é dito monomorfismo essencial quando, para qualquer $f: M \rightarrow N$, vale:

$$
f g \text { é monomorfismo } \Rightarrow f \text { é monomorfismo. }
$$

Dada a álgebra de $\operatorname{Artin} \Lambda$, se $M \in \bmod (\Lambda)$, sempre existe e é única, a menos de isomorfismos, a cobertura projetiva de $M$, ou seja, sempre existe um epimorfismo essencial $f: P_{0} \rightarrow M$ com $P_{0}$ um $\Lambda$-módulo projetivo finitamente gerado. Para aquele $M$, sempre existe também sua envolvente injetiva $\mathrm{I}_{0}(M)$, isto é, um monomorfismo essencial $\mathrm{g}: M \rightarrow$ $\mathrm{I}_{0}(M) \operatorname{com} \mathrm{I}_{0}(M) \in \bmod (\Lambda)$ um injetivo.

Uma seqüência de morfismos $L \stackrel{f}{\rightarrow} M \stackrel{g}{\rightarrow} N$ é uma seqüência exata em $M$ quando $\operatorname{ker}(g)=\operatorname{im}(f)$. Uma seqüência de morfismos

$$
\ldots \rightarrow M_{t} \rightarrow M_{t+1} \rightarrow M_{t+2} \rightarrow \ldots
$$

é uma seqüência exata quando, para cada $i$, a seqüência $M_{i-1} \rightarrow M_{i} \rightarrow M_{i+1}$ é exata. 
Uma resolução projetiva de um ^-módulo $M$ é uma seqüência exata (finita à esquerda ou não)

$$
\cdots \rightarrow P_{3} \stackrel{f_{3}}{\rightarrow} P_{2} \stackrel{f_{2}}{\rightarrow} P_{1} \stackrel{f_{1}}{\rightarrow} P_{0} \stackrel{f_{0}}{\rightarrow} M \rightarrow 0
$$

onde $P_{\mathfrak{i}} \in \bmod (\Lambda)$ é um projetivo. Tal resolução é dita minimal quando $f_{0}: P_{0} \rightarrow M$ é uma cobertura projetiva de $M$ e, para cada $j, P_{j} \stackrel{f_{j}}{\rightarrow} \operatorname{im}\left(f_{j}\right)$ é uma cobertura projetiva de $\operatorname{im}\left(f_{j}\right)=\operatorname{ker}\left(f_{j-1}\right)$. Se a seqüência é finita à esquerda com $P_{r}$ seu último termo não nulo, diz-se que a resolução tem comprimento $r+1$.

$\mathrm{O} \wedge$-módulo $M$ tem dimensão projetiva menor ou igual a $r$ (em símbolos, $\operatorname{pd}(M) \leqslant r$ ) quando existe uma resolução projetiva minimal de $M$, com comprimento $r+1$. Se toda resolução projetiva minimal de $M$ for infinita, diz-se que $\operatorname{pd}(M)=\infty$. Se $n$ é o menor natural tal que $\operatorname{pd}(M) \leqslant n$ então $\operatorname{pd}(M)=n$.

Uma resolução injetiva de $M$ é uma seqüência exata

$$
0 \rightarrow M \stackrel{f_{0}}{\rightarrow} I_{0} \stackrel{f_{1}}{\rightarrow} I_{1} \stackrel{f_{2}}{\rightarrow} I_{2} \stackrel{f_{3}}{\longrightarrow} \ldots
$$

onde $I_{i} \in \bmod (\Lambda)$ é um injetivo. Tal resolução é dita minimal quando $M \stackrel{f_{0}}{\rightarrow} I_{0}$ é uma envolvente injetiva de $M$ e, para cada $j$, o morfismo $f_{j}: \operatorname{coker}\left(f_{j-1}\right) \rightarrow I_{j}$ for uma envolvente injetiva de $\operatorname{coker}\left(f_{j-1}\right)=\frac{I_{j}}{\operatorname{im}\left(f_{j}\right)}$.

$\mathrm{O} \wedge$-módulo $M$ tem dimensão injetiva menor ou igual a $r$ (em símbolos, $\operatorname{id}(M) \leqslant r$ ) quando existe uma resolução injetiva minimal de $M$ com comprimento $r+1$. Se toda resolução injetiva minimal de $M$ for infinita, diz-se que $\operatorname{id}(M)=\infty$. Se $n$ é o menor natural tal que id $(M) \leqslant n$ então $\operatorname{id}(M)=n$

Observe que todo $\Lambda$-módulo finitamente gerado admite resoluções injetivas e projetivas. Este fato e o teorema que segue após a definição abaixo pode ser esclarecido no capítulo X em [Ass97].

1.7 Definição. A dimensão global de uma álgebra de Artin $\Lambda$ é o número $n=\sup \{\operatorname{pd}(M): M \in \bmod (\Lambda)\} . \operatorname{Notação:~} n=\operatorname{gl} \cdot \operatorname{dim}(\Lambda)$.

1.8 Teorema. Para uma álgebra de Artin $\wedge$, valem as igualdades: $\operatorname{gl} \operatorname{dim}(\Lambda)=\sup \{\operatorname{pd}(M): M \in \bmod (\Lambda)\}=\sup \{\operatorname{id}(M): M \in \bmod (\Lambda)\}$

O próximo lema, usado mais a frente, é conhecido como Lema de Schanuel. Por não ter sido encontrada uma demonstração para a versão deste lema usada nesta dissertação, optou-se por demonstrá-lo no fim desta seção. 
1.9 Lema. Considere as seqüências exatas abaixo, onde os termos do meio são injetivos.

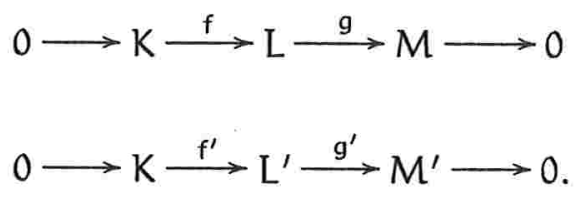

Então, $\mathrm{M} \oplus \mathrm{L}^{\prime} \cong \mathrm{L} \oplus \mathrm{M}^{\prime}$.

Demonstração. Não é demasiada restrição considerar $\mathrm{K} \subset \mathrm{L}$ e $\mathrm{K} \subset \mathrm{L}^{\prime}$, uma vez que $\operatorname{im}(f) \cong K \cong \operatorname{im}\left(f^{\prime}\right)$.

Considere o módulo $L \oplus L^{\prime}$ e seu submódulo $A=\left\{(a, a) \in L \oplus L^{\prime}: a \in K\right\}$.

A aplicação $h$ definida abaixo é um epimorfismo, já que dado $x^{\prime} \in L^{\prime}$, vale $h\left(0, x^{\prime}\right)=x^{\prime}$.

$$
\begin{aligned}
\mathrm{h}: \mathrm{K} \oplus \mathrm{L}^{\prime} & \rightarrow \mathrm{L}^{\prime} \\
\left(\mathrm{a}, \mathrm{l}^{\prime}\right) & \mapsto \mathrm{l}^{\prime}-\mathrm{a}
\end{aligned}
$$

Observe que o kernel de $h$ é $A$, pois $h\left(a, l^{\prime}\right)=0 \Leftrightarrow l^{\prime}-a=0 \Leftrightarrow l^{\prime}=a \Leftrightarrow l^{\prime} \in K$. Então, do bem conhecido teorema do homomorfismo ([AF92, 3.7]), o módulo $V^{\prime}=\frac{K \oplus L^{\prime}}{A}$ é isomorfo a $L^{\prime}$. Escrevendo $W=\frac{L \oplus L^{\prime}}{A}$, tem-se

$$
\frac{W}{V^{\prime}} \cong \frac{L \oplus L^{\prime}}{K \oplus L^{\prime}} \cong \frac{L}{K} \cong M
$$

Como $\mathrm{V}^{\prime} \cong \mathrm{L}^{\prime}$ é um injetivo, é somando de todo módulo que o contém. Então, existe um complementar $C^{\prime}$ tal que $V^{\prime} \oplus C^{\prime}=W$. E daí, $C^{\prime} \cong \frac{W}{V^{\prime}} \cong M$. Portanto,

$$
W \cong V^{\prime} \oplus C^{\prime} \cong L^{\prime} \oplus M
$$

Alterando convenientemente a aplicação $h$ acima, um argumento semelhante mostra que $V^{\prime \prime} .=\frac{K \oplus L}{A} \cong L$. E então, existe $C^{\prime \prime}$ tal que $V^{\prime \prime} \oplus C^{\prime \prime} \cong W$. Como $\frac{W}{V^{\prime \prime}} \cong M^{\prime}$, tem-se $C^{\prime \prime} \cong \frac{W}{V^{\prime \prime}} \cong M^{\prime} . \mathrm{E}$ assim,

$$
W \cong V^{\prime \prime} \oplus C^{\prime \prime} \cong L \oplus M^{\prime}
$$

Assim, das equações (1.1) e (1.2), vale

$\mathrm{L} \oplus \mathrm{M}^{\prime} \cong \mathrm{V}^{\prime \prime} \oplus \mathrm{C}^{\prime \prime} \cong W \cong \mathrm{V}^{\prime} \oplus \mathrm{C}^{\prime} \cong \mathrm{L}^{\prime} \oplus M$. 


\subsection{Algumas equivalências}

Esta seção contém alguns resultados importantes, para os quais uma demonstração mais geral pode ser obtida. Nesta dissertação, serão tomadas subcategorias da categoria de módulos sobre álgebras de Artin.

Seja $\mathcal{C}$ uma subcategoria plena de $\bmod (\Lambda)$.

Uma $\mathcal{C}$-apresentação do $\Lambda$-módulo $M$ é uma seqüência exata $\mathrm{Q}_{1} \stackrel{\mathrm{f}_{1}}{\rightarrow} \mathrm{Q}_{0} \stackrel{\mathrm{f}_{0}}{\rightarrow} \mathrm{M} \rightarrow 0$, com $Q_{1}$ e $Q_{0}$ em $\mathcal{C}$. Uma $\mathcal{C}$-coapresentação do $\wedge$-módulo $M$ é uma seqüência exata $0 \rightarrow M \stackrel{f_{0}}{\rightarrow} Q_{0} \stackrel{f_{1}}{\rightarrow} Q_{1}$, com $Q_{1}$ e $Q_{0}$ em $\mathcal{C}$.

Seja $M \in \bmod (\Lambda)$. Denota-se por $\mathcal{P}_{1}^{M}$ a subcategoria plena de $\bmod (\Lambda)$ formada por todos os $\Lambda$-módulos que admitem uma $\operatorname{add}(M)$-apresentação, e $J_{1}^{M}$ denota a subcategoria plena de $\bmod (\Lambda)$ formada por todos os $\Lambda$-módulos que admitem uma $\operatorname{add}(M)$ coapresentação. Estas categorias, $\mathcal{P}_{1}^{M}$ e $\mathcal{J}_{1}^{M}$, são importantes quando deseja-se encontrar equivalências entre categorias de módulos, conforme se vê na demonstração do teorema de Morita mais abaixo.

Obșerve que se $M \in \bmod (\Lambda)$, então a Proposição 1.4 diz que $\operatorname{End}_{\Lambda}(M)$ é uma álgebra de Artin, e portanto, $\Gamma=\operatorname{End}_{\Lambda}(M)^{\text {op }}$ também é álgebra de Artin. Esta observação é importante ao enunciar o lema abaixo, que estabelece equivalências entre certas categorias. A demonstração da primeira afirmação pode ser observada em [ARS95], capítulo II, 2.5. A segunda tem prova dual.

1.10 Lema. Sejam $\wedge$ uma álgebra de Artin, P um ^-módulo projetivo e I um $\wedge$-módulo injetivo. Considere $\Gamma=\operatorname{End}_{\Lambda}(\mathrm{P})^{\mathrm{op}}$ e $\Gamma^{\prime}=\operatorname{End}_{\Lambda}(\mathrm{I})^{\mathrm{op}}$. Então os funtores

$$
\mathrm{X} \in \mathcal{P}_{1}^{\mathrm{P}} \mapsto \operatorname{Hom}_{\wedge}(\mathrm{P}, \mathrm{X}) \in \bmod (\Gamma) \text { e } \mathrm{X} \in \mathcal{J}_{1}^{\mathrm{I}} \mapsto \operatorname{Hom}_{\wedge}(\mathrm{X}, \mathrm{I}) \in \bmod \left(\Gamma^{\prime}\right)
$$

são equivalências entre categorias.

Observação 1.11. Se $\wedge$ está em add(P), para algum projetivo $\mathrm{P}$, então todo projetivo indecomponível está em add(P), pela Proposição 1.6. Neste caso, se $M \in \bmod (\Lambda)$, então uma resolução projetiva de $M$, digamos

$$
\cdots \rightarrow P_{3} \stackrel{f_{3}}{\rightarrow} P_{2} \stackrel{f_{2}}{\rightarrow} P_{1} \stackrel{f_{1}}{\rightarrow} P_{0} \stackrel{f_{0}}{\rightarrow} M \rightarrow 0,
$$

fornece uma add( $\mathrm{P})$-apresentação, já que $\mathrm{P}_{0}$ e $\mathrm{P}_{1}$ são somas de projetivos indecomponíveis, e portanto, estão em add(P). 
Logo, pode-se concluir que se $\Lambda \in \operatorname{add}(P)$, com $P$ projetivo, então $\bmod (\Lambda)=\mathcal{P}_{1}^{P}$.

1.12 Definição. Duas álgebras de Artin $\Lambda$ e $\Lambda^{\prime}$ são Morita-equivalentes quando existe uma equivalência entre as categorias de $\operatorname{módulos} \bmod (\Lambda)$ e $\bmod \left(\Lambda^{\prime}\right)$. A notação, neste caso, é $\wedge \simeq_{m} \wedge^{\prime}$.

Observe que a relação $\Lambda \simeq_{m} \Lambda^{\prime}$ define sobre a classe das álgebras de Artin uma relação de equivalência.

Estamos em condições de enunciar e demonstrar o Teorema de Morita. Este teorema ajuda a decidir quando duas álgebras são Morita-equivalentes. A notação $\mathcal{A} \equiv \mathcal{C}$ indica que há uma equivalência entre as categorias $\mathcal{A}$ e $\mathcal{C}$, enquanto a notação $\mathrm{A} \cong \mathrm{B}$ indica que os anéis $A$ e $B$ são isomorfos.

1.13 Teorema. As afirmações abaixo são equivalentes, para álgebras de Artin $\Lambda$ e $\wedge^{\prime}$.

1. $\wedge \simeq_{m} \wedge^{\prime}$

2. Existe um $\Lambda$-módulo projetivo finitamente gerado $\mathrm{P}$ tal que $\Lambda \in \operatorname{add}(\mathrm{P}) e \operatorname{End}_{\Lambda}(\mathrm{P}) \cong \Lambda^{\prime o p}$ como anéis.

3. Existem projetivos $\mathrm{P} \in \bmod (\Lambda), \mathrm{Q} \in \bmod \left(\Lambda^{\prime}\right)$ tais que $\Lambda \in \operatorname{add}(\mathrm{P}), \Lambda^{\prime} \in \operatorname{add}(\mathrm{Q})$ $e \operatorname{End}_{\Lambda}(\mathrm{P}) \cong \operatorname{End}_{\Lambda^{\prime}}(\mathrm{Q})$

Demonstração.

$(1 \Rightarrow 2)$

Seja $F: \bmod \left(\Lambda^{\prime}\right) \rightarrow \bmod \Lambda$ uma equivalência e faça $P=F\left(\Lambda^{\prime}\right)$.

Aplicando o Teorema de Krull-Schmidt ao $\Lambda$-módulo projetivo $\Lambda^{\prime}$, tem-se $\Lambda^{\prime}=\oplus \mathrm{P}_{j}^{\prime}$. Portanto $P=F\left(\Lambda^{\prime}\right)=F\left(\oplus P_{j}^{\prime}\right)=\oplus F\left(P_{j}^{\prime}\right)$.

Considere $P_{i}$ um projetivo indecomponível em $\bmod (\Lambda)$ e recorde que $F$ é denso. Então existe um projetivo indecomponível $\mathrm{P}_{\mathrm{j}_{\mathrm{i}}}^{\prime}$ em $\bmod \left(\Lambda^{\prime}\right)$ tal que $\mathrm{F}\left(\mathrm{P}_{\mathrm{j}_{\mathrm{i}}}^{\prime}\right)=\mathrm{P}_{\mathrm{i}}$.

Daí, $\Lambda=\oplus \mathrm{P}_{\mathrm{i}}=\oplus \mathrm{F}\left(\mathrm{P}_{\mathrm{j}_{\mathrm{i}}}^{\prime}\right)$. Mas $\oplus \mathrm{F}\left(\mathrm{P}_{\mathrm{j}_{\mathrm{i}}}^{\prime}\right)$ é somando direto de $\oplus \mathrm{F}\left(\mathrm{P}_{\mathrm{j}}^{\prime}\right)=\mathrm{P}$. Portanto $\Lambda \in \operatorname{add}(P)$. 
Observe também que, por ser F um funtor plenamente fiel, vale

$$
\operatorname{End}_{\Lambda}(P)=\operatorname{Hom}_{\Lambda}\left(F\left(\Lambda^{\prime}\right), F\left(\Lambda^{\prime}\right)\right) \cong \operatorname{Hom}_{\Lambda}\left(\Lambda^{\prime}, \Lambda^{\prime}\right) \cong\left(\Lambda^{\prime}\right)^{o p}
$$

onde os isomorfismos são isomorfismos de anéis.

$(2 \Rightarrow 3)$

Seja $\mathrm{Q}=\wedge^{\prime} \Lambda^{\prime}$. Então, $\mathrm{Q}$ é $\Lambda$-módulo projetivo finitamente gerado. É claro que $\wedge^{\prime} \Lambda^{\prime} \in \operatorname{add}(Q)$.

Ora, $\Lambda^{\prime o p} \cong \operatorname{End}_{\Lambda^{\prime}}\left(\Lambda^{\prime}\right)$ como anéis.

Então, por hipótese, $\operatorname{End}_{\wedge}(P) \cong \operatorname{End}_{\Lambda^{\prime}}(Q) \cong \Lambda^{\prime o p}$.

$(3 \Rightarrow 1)$ Considere as álgebras de $\operatorname{Artin} \Gamma=\operatorname{End}_{\Lambda}(P)^{o p}$ e $\Gamma^{\prime}=\operatorname{End}_{\Lambda^{\prime}}(Q)^{o p}$.

Por hipótese, $\Gamma^{\mathrm{op}} \cong \Gamma^{\prime \mathrm{op}}$ como anéis: Então $\Gamma \cong \Gamma^{\prime}$. Neste caso, $\bmod (\Gamma) \operatorname{e} \bmod \left(\Gamma^{\prime}\right)$ são equivalentes.

Do lema 1.10, por serem $P$ e $Q$ projetivos, $\mathcal{P}_{1}^{P}$ é equivalente a $\bmod (\Gamma)$, bem como $\mathcal{P}_{1}^{Q}$ é equivalente a $\bmod \left(\Gamma^{\prime}\right)$.

Como $\Lambda \in \operatorname{add}(P), \Lambda^{\prime} \in \operatorname{add}(Q)$, então a Observação $1.11 \operatorname{diz}$ que $\mathcal{P}_{1}^{P}=\bmod (\Lambda) \mathrm{e}$ $\mathrm{P}_{1}^{\mathrm{Q}}=\bmod \left(\Lambda^{\prime}\right)$.

Assim,

$\bmod (\Lambda)=\mathcal{P}_{1}^{P} \equiv \bmod (\Gamma) \equiv \bmod \left(\Gamma^{\prime}\right) \equiv \mathcal{P}_{1}^{\mathrm{Q}}=\bmod \left(\Lambda^{\prime}\right)$ conforme enunciado.

\subsection{Funtores Coerentes}

O interesse maior desta seção é estabelecer algumas definições e enunciar resultados sobre certas categorias de funtores, que serão relevantes no próximo capítulo.

1.14 Definição. Uma categoria C é uma categoria aditiva quando são satisfeitas as seguintes condições:

1. Para cada par de objetos $X$ e $Y$, o conjunto $\operatorname{Hom}_{\mathcal{C}}(X, Y)$ é um grupo abeliano.

2. Para cada par de objetos $\mathrm{X}$ e $\mathrm{Y}$, existe uma soma direta $\mathrm{X} \oplus \mathrm{Y}=\mathrm{M} \in \mathcal{C}$, ou seja, existe um objeto $M$ e morfismos $i_{1}$ e $i_{2}, \pi_{1}$ e $\pi_{2}$

$$
X \underset{\pi_{1}}{\stackrel{i_{1}}{\rightleftarrows}} X \oplus Y \stackrel{\pi_{2}}{\underset{i_{2}}{\rightleftarrows}} Y
$$


tais que

- $\pi_{1} i_{1}=I_{X}$

- $\pi_{2} i_{2}=I_{Y}$

- $i_{1} \pi_{1}+i_{2} \pi_{2}=I_{X \oplus Y}$

onde $\mathrm{I}_{\mathrm{X}}$ indica o morfismo identidade em $\mathrm{X}$.

3. A composição de morfismos em $\mathcal{C}$ é $\mathbb{Z}$-bilinear, ou seja $(f+g) h=f h+g h$ e $f(g+h)=f g+f h$ para quaisquer morfismos $f, g$ e $h$ de $\mathcal{C}$.

\subsection{Exemplos.}

a) Seja Ab a categoria cujos objetos são os grupos abelianos e cujos morfismos são os homomorfismos de grupo, com composição de funções.

Então Ab é uma categoria aditiva, pois, para cada par de grupos abelianos $\mathrm{X}$ e $\mathrm{Y}$, e para cada par de morfismos $f$ e $g$ em $\operatorname{Hom}(X, Y)$, definindo $(f+g)(x)=f(x)+g(x)$, o conjunto $\operatorname{Hom}(X, Y)$ torna-se um grupo abeliano.

A soma direta será, para dois grupos abelianos $X$ e $Y$, o grupo abeliano $X \oplus Y=X \times Y$, produto cartesiano de $\mathrm{X}$ e $\mathrm{Y}$, com operação induzida pelas operações em $\mathrm{X}$ e $\mathrm{Y}$.

b) De maneira semelhante, se $\Lambda$ é álgebra de $\operatorname{Artin}, \bmod (\Lambda)$ também é categoria aditiva, uma vez que, dados os módulos $M$ e $N, \operatorname{Hom}_{\wedge}(M, N)$ é grupo abeliano e a soma direta $M \oplus N$ de módulos satisfaz as propriedades acima.

c) Seja $M \in \bmod (\Lambda)$, onde $\Lambda$ é uma álgebra de Artin. A categoria add $(M)$ é categoria aditiva. Com efeito, por ser $\operatorname{add}(M)$ uma subcategoria plena de $\bmod (\Lambda)$, há a soma direta de objetos de $\operatorname{add}(M)$ e esta soma direta está em $\operatorname{add}(M)$.

1.16 Definição. Um funtor $F: \mathcal{C} \rightarrow \mathcal{D}$ entre duas categorias aditivas $\mathcal{C}$ e $\mathcal{D}$ é funtor aditivo quando, para cada par de objetos $\mathrm{X}$ e $\mathrm{Y}$, a aplicação

$$
\begin{array}{cccc}
\operatorname{Hom}(X, Y) & \rightarrow & \operatorname{Hom}(F(X), F(Y)) \\
h & \mapsto & F(h)
\end{array}
$$

é homomorfismo de grupos abelianos. 
Para dar um exemplo de funtor aditivo, considere uma álgebra de Artin $\wedge$ e $A \in \bmod (\Lambda)$ fixado. Então o funtor

$$
\begin{aligned}
\operatorname{Hom}_{\Lambda}(A,-): \bmod (\Lambda) & \rightarrow \quad \mathrm{Ab} \\
B & \mapsto \operatorname{Hom}_{\Lambda}(A, B)
\end{aligned}
$$

é funtor aditivo.

Lembre que, para o morfismo $f: B_{1} \rightarrow B_{2} \operatorname{em} \bmod (\Lambda)$, está bem definido o morfismo em $\mathrm{Ab}$

$$
\begin{aligned}
\operatorname{Hom}(A, f): & \operatorname{Hom}\left(A, B_{1}\right) \rightarrow \operatorname{Hom}\left(A, B_{2}\right) \\
g: A \rightarrow B_{1} & \mapsto f g: A \rightarrow B_{2}
\end{aligned}
$$

Considere agora $\mathcal{C}$ uma categoria aditiva. Seja $A$ um objeto de $\mathcal{C}$ fixado. Denote por $\left[\operatorname{Hom}_{\mathcal{C}}(A,-), F\right]$ a coleção de aplicações naturais entre os funtores aditivos $\mathrm{F}: \mathcal{C} \rightarrow \mathbf{A b} \mathrm{e}$ $\operatorname{Hom}_{\mathcal{C}}(A,-): \mathcal{C} \rightarrow \mathbf{A b}$.

O lema seguinte, adaptação do tradicional Lema de Yoneda, é uma ferramenta útil e uma demonstração completa pode ser encontrada em [Jac89]. Neste texto, tanto este Lema quanto a observação que o segue merecem atenção.

1.17 Lema (de Yoneda). A coleção de aplicações naturais $\left[\operatorname{Hom}_{\mathbb{C}}(\mathrm{A},-), \mathrm{F}\right]$ é um grupo abeliano, isomorfo a $\mathrm{F}(\mathrm{A})$.

Demonstração. De fato, são aplicações inversas uma da outra

$$
\begin{array}{cl}
\mathfrak{F}:\left[\operatorname{Hom}_{\mathcal{C}}(A,-), \mathrm{F}\right] & \rightarrow \mathrm{F}(A) \\
\varphi & \mapsto \mathfrak{F}(\varphi)=\varphi_{A}\left(\mathrm{I}_{\mathrm{A}}\right)
\end{array}
$$

onde $I_{A}$ indica o morfismo identidade de $A$ e

$$
\begin{aligned}
\mathfrak{G}: \mathrm{F}(A) & \rightarrow\left[\operatorname{Hom}_{\mathcal{e}}(A,-), \mathrm{F}\right] \\
\mathrm{a} & \mapsto \mathfrak{G}(\mathrm{a})=\varphi_{\mathrm{a}}
\end{aligned}
$$

onde $\varphi_{\mathrm{a}}$ é a aplicação natural

$$
\varphi_{a}=\left\{\begin{aligned}
{\left[\varphi_{a}\right]_{X}: } & \operatorname{Hom}_{\mathcal{C}}(A, X) \rightarrow F(X) \\
(k: A \rightarrow X) & \mapsto F(k)(a)
\end{aligned}\right\}_{X \in \mathcal{C}} .
$$

Isto basta para mostrar que há uma bijeção entre $\left[\operatorname{Hom}_{\mathcal{C}}(A,-), F\right]$ e $F(A)$. Para completar a prova do lema, é necessário munir o conjunto $\left[\operatorname{Hom}_{\mathcal{C}}(A,-), F\right]$ com uma operação 
para torná-lo grupo abeliano; se $\varphi$ e $\psi$ são aplicações naturais em $\left[\operatorname{Hom}_{\mathcal{C}}(A,-), F\right]$, então a adição

$$
\varphi+\psi=\left\{\begin{aligned}
{[\varphi+\psi]_{X}: } & \operatorname{Hom}_{\mathcal{C}}(A, X) \rightarrow F(X) \\
(k: A \rightarrow X) & \mapsto \varphi_{X}(k)+\psi_{X}(k)
\end{aligned}\right\}_{X \in \mathcal{C}}
$$

faz de $\left[\operatorname{Hom}_{\mathcal{C}}(A,-), F\right]$ um grupo abeliano, tendo como elemento neutro a aplicação natural

$$
0=\left\{\begin{aligned}
0_{X}: \operatorname{Hom}_{\mathcal{C}}(A, X) & \rightarrow F(X) \\
(k: A \rightarrow X) & \mapsto 0
\end{aligned}\right\}_{X \in \mathcal{C}}
$$

Para finalizar, observe que, para aquelas $\varphi$ e $\psi$, vale

$$
\mathfrak{F}(\varphi+\psi)=(\varphi+\psi)_{A}\left(I_{A}\right) \doteq \varphi_{A}\left(I_{A}\right)+\psi_{A}\left(I_{A}\right)=\mathfrak{F}(\varphi)+\mathfrak{F}(\psi),
$$

o que mostra que $\mathfrak{F}$ é homomorfismo de grupos abelianos.

Observação 1.18. Vale uma versão deste lema para funtores contravariantes, ou seja, se F é um funtor contravariante, então a coleção $\left[\operatorname{Hom}_{\mathcal{C}}(-, A), F\right]$ é um grupo abeliano, isomorfo a $F(A)$.

1.19 Exemplo. Se $F=\operatorname{Hom}_{\mathcal{C}}\left(-, A^{\prime}\right)$ para algum objeto $A^{\prime}$, então são isomorfos como grupos abelianos $\left[\operatorname{Hom}_{\mathfrak{e}}(-, A), \operatorname{Hom}_{\mathfrak{e}}\left(-, A^{\prime}\right)\right]$ e $\operatorname{Hom}_{\mathfrak{e}}\left(A, A^{\prime}\right)$.

A próxima definição aparece de uma maneira mais geral em trabalhos de Auslander, anteriores àquele estudado por esta dissertação ([Aus71]). Veja por exemplo [Aus66]. Atualmente, uma abordagem moderna chama os funtores coerentes de funtores finitamente gerados, por haver uma disposição em comparar categorias de funtores com categorias de módulos (veja por exemplo [APT]). Neste trabalho opta-se por manter a terminologia original.

1.20 Definição. Seja $M$ um módulo finitamente gerado sobre uma álgebra de Artin. Um funtor aditivo $\mathrm{F}: \operatorname{add}(M) \rightarrow \mathrm{Ab}$ é chamado funtor coerente quando existe um morfismo $f: A_{1} \rightarrow A_{2}$ em $\operatorname{add}(M)$ tal que, para qualquer $X$ em add $(M)$, a seqüência de grupos abelianos abaixo é exata :

$$
\operatorname{Hom}_{\mathcal{C}}\left(X, A_{1}\right) \stackrel{\operatorname{Hom}(X, f)}{\longrightarrow} \operatorname{Hom}_{\mathcal{C}}\left(X, A_{2}\right) \rightarrow F(X) \rightarrow 0
$$


1.21 Exemplo. Seja $X \in \bmod (\Lambda)$. Denota-se por $(-, X)$ a restrição do funtor $\operatorname{Hom}(-, X): \bmod (\Lambda) \rightarrow \mathbf{A b}$ à subcategoria $\operatorname{add}(M)$. Assim, o objeto resultante da aplicação do funtor $(-, X)$ sobre um objeto $T \in \operatorname{add}(M)$ é o objeto $(T, X)$.

Então o funtor $(-, X): \operatorname{add}(M) \rightarrow$ Ab é um exemplo de funtor coerente.

1.22 Exemplo. Seja $f: I_{0} \rightarrow I_{1}$ um morfismo em add(M). Então considere o funtor

$$
F=(-, \operatorname{coker}(f)): \operatorname{add}(M) \rightarrow A b
$$

cuja associação entre objetos é dada pela igualdade

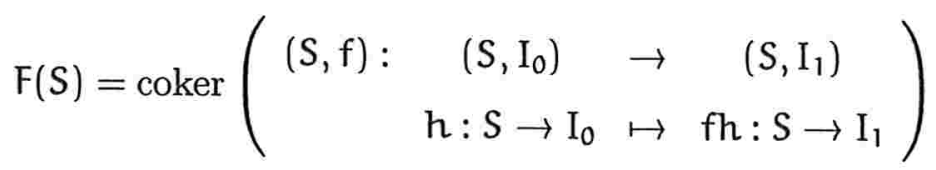

e que, a cada morfismo $\mathrm{t}: \mathrm{J} \rightarrow \mathrm{N}$ em add( $M)$, associa o morfismo

$$
\begin{aligned}
\tau_{\mathrm{t}}= & \operatorname{coker}(\mathrm{N}, \mathrm{f}) \rightarrow \operatorname{coker}(\mathrm{J}, \mathrm{f}) \\
{\left[\mathrm{h}: \mathrm{N} \rightarrow \mathrm{I}_{1}\right] } & \mapsto\left[\mathrm{ht}: \mathrm{J} \rightarrow \mathrm{I}_{1}\right]
\end{aligned}
$$

onde os morfismos entre colchetes são representantes das classes de equivalência nos cokernels.

Observe que $\tau_{t}$ está bem definido.

Então $F$ é um exemplo de funtor coerente, pois é exata em $\mathbf{A b}$, para cada $X \in \operatorname{add}(M)$ a seqüência

$$
\left(X, I_{0}\right) \stackrel{(X, f)}{\longrightarrow}\left(X, I_{1}\right) \rightarrow F(X) \rightarrow 0
$$

Se $M \in \bmod (\Lambda)$, denota-se por $\mathcal{F}_{M}$ a coleção de funtores coerentes $F: \operatorname{add}(M) \rightarrow \mathbf{A b}$. Esta coleção admite estrutura de categoria quando se considera como morfismos entre dois funtores $\mathrm{F}$ e $\mathrm{G}$ as aplicações naturais $\varphi: \mathrm{F} \rightarrow \mathrm{G}$. É com a estrutura de categoria que $\mathcal{F}_{M}$ será apontada.

O próximo lema, além de ser importante em demonstrações no próximo capítulo, permitịá neste momento definir um conceito de seqüência exata na categoria $\mathcal{F}_{M}{ }^{1}$

\footnotetext{
${ }^{1}$ A definição dada concorda com a definição mais geral que se dá para categorias abelianas, como em [Aus71], evitando desenvolver a parte da teoria de categorias necessária para definir as tais seqüências exatas em categorias abelianas.
} 
Este lema estabelece uma equivalência entre a categoria de funtores coerentes e uma certa categoria de módulos associada. Sua demonstração envolve conceitos categóricos e homológicos que fogem ao escopo dessa dissertação e pode ser encontrada no artigo original de M. Auslander em [Aus71, 3.2]

1.23 Lema. Seja $\wedge$ uma álgebra de Artin e $M \in \bmod (\Lambda)$ um módulo tal que $\Lambda \in \operatorname{add}(M)^{2}$. Considere a álgebra de Artin $\Gamma=\operatorname{End}_{\Lambda}(M)^{o p}$. O funtor

$$
\begin{aligned}
\mathfrak{F}: \mathcal{F}_{M} & \rightarrow \bmod (\Gamma) \\
\mathrm{F} & \mapsto \mathrm{F}(\mathrm{M})
\end{aligned}
$$

é uma equivalência entre $\mathcal{F}_{M}$ e a categoria de $\Gamma$-módulos à direita, finitamente gerados.

1.24 Definição. Uma seqüência de aplicações naturais $F_{1} \stackrel{\varphi}{\rightarrow} F_{2} \stackrel{\Psi}{\rightarrow} F_{3}$ em $\mathcal{F}_{M}$ é uma seqüência exata em $\mathcal{F}_{M}$ quando a seqüência $F_{1}(M) \stackrel{\varphi_{M}}{\longrightarrow} F_{2}(M) \stackrel{\psi_{M}}{\longrightarrow} F_{3}(M)$ é exata em $\bmod (\Gamma)$.

A definição acima admite a seguinte forma equivalente:

1.25 Proposição. A seqüência $\mathrm{F}_{1} \stackrel{\varphi}{\rightarrow} \mathrm{F}_{2} \stackrel{\Psi}{\rightarrow} \mathrm{F}_{3}$ é exata em $\mathcal{F}_{M}$ se e somente se, para cada $C \in \operatorname{add}(M)$, a seqüência $F_{1}(C) \stackrel{\varphi_{C}}{\longrightarrow} F_{2}(C) \stackrel{\psi_{C}}{\longrightarrow} F_{3}(C)$ é exata em $\bmod (\Gamma)$.

Observação 1.26. Recorde que, em uma categoria $\mathcal{C}$, um objeto $\mathrm{P}$ é chamado objeto projetivo exatamente da mesma maneira como para módulos, ou seja, quando, para cada epimorfismo $g: M \rightarrow N$ e para cada morfismo $f: P \rightarrow N$, existe um morfismo $f^{\prime}: P \rightarrow M$ tal que $g f^{\prime}=f$.

De maneira análoga, um objeto I é um objeto injetivo quando, para cada monomorfismo $g: N \rightarrow M$, e para cada $f: N \rightarrow I$, existe $f^{\prime}: M \rightarrow I$ tal que $f^{\prime} g=f$.

Quando faz sentido falar de seqüências exatas em uma categoria $\mathcal{C}$, as definições de dimensão projetiva e dimensão injetiva para um objeto $\mathrm{C}$ são semelhantes às dadas para módulos, de modo que não serão explicitadas. As notações, neste caso, será $\operatorname{pd}_{\mathcal{C}}(C)$ e $\operatorname{ide}_{e}(C)$.

Assim também será feito para a definição de dimensão global da categoria $\mathcal{C}$, que será denotada por gl. $\operatorname{dim}(\mathcal{C})$.

\footnotetext{
${ }^{2}$ tal módulo será posteriormente chamado de gerador de $\bmod (\Lambda)$
} 
Observação 1.27. Note que a equivalência estabelecida no Lema 1.23 garante que gl. $\operatorname{dim}\left(\mathcal{F}_{M}\right)=$ gl. $\operatorname{dim}(\Gamma)$.

Convém, neste momento, descrever os objetos projetivos da categoria $\mathcal{F}_{M}$. A próxima proposição mostra exemplos de projetivos em $\mathcal{F}_{M}$ e também mostra como são todos os projetivos da categoria $\mathcal{F}_{M}$.

1.28 Proposição. São verdadeiras as afirmações:

a) $O$ funtor $(-, C): \operatorname{add}(M) \rightarrow$ Ab é projetivo em $\mathcal{F}_{M}$, para todo $C \in \operatorname{add}(M)$;

b) Se $\mathrm{F}$ é projetivo em $\mathcal{F}_{\mathrm{M}}$, então existe $\mathrm{C} \in \operatorname{add}(\mathrm{M})$ tal que $\mathrm{F} \cong(-, \mathrm{C})$.

Demonstração.

a) Basta provar que, para cada seqüência exata $F_{1} \stackrel{\varphi}{\rightarrow} F_{2} \stackrel{\Psi}{\rightarrow} F_{3}$ em $\mathcal{F}_{M}$, é exata a seqüência em Ab:

$$
\left((-, C), F_{1}\right) \stackrel{((-, C), \varphi)}{\longrightarrow}\left((-, C), F_{2}\right) \stackrel{((-, C), \psi)}{\longrightarrow}\left((-, C), F_{3}\right)
$$

Se $F_{1} \stackrel{\varphi}{\rightarrow} F_{2} \stackrel{\Psi}{\rightarrow} F_{3}$ é exata em $\mathcal{F}_{M}$, do lema anterior, $F_{1}(C) \stackrel{\varphi_{C}}{\longrightarrow} F_{2}(C) \stackrel{\psi_{C}}{\longrightarrow} F_{3}(C)$ é exata em $\bmod (\Gamma)$. Mas o Lema 1.18 diz que $F_{i}(C) \cong\left((-, C), F_{i}\right)$. Assim, através dos morfismos $\mathfrak{F}$ e $\mathfrak{G}$ definidos no Lema 1.17, é exata a seqüência

$$
\left((-, C), F_{1}\right) \stackrel{\mathfrak{B} \varphi_{\mathrm{C}} \mathfrak{F}}{\longrightarrow}\left((-, C), F_{2}\right) \stackrel{\mathfrak{B} \psi_{\mathrm{C}} \mathfrak{F}}{\longrightarrow}\left((-, C), F_{3}\right)
$$

Observe por fim que os morfismos $\mathfrak{G} \varphi_{\mathrm{C}} \mathfrak{F}$ e $((-, \mathrm{C}), \varphi)$ são iguais, bem como são iguais os morfismos $\mathfrak{G} \psi_{\mathrm{C}} \mathfrak{F}$ e $((-, C), \psi)$. Isso termina essa parte da demonstração.

b) Com efeito, observe que se $F$ é coerente, há um morfismo $A_{1} \stackrel{f}{\rightarrow} A_{2}$ tal que

$$
\left(-, A_{1}\right) \stackrel{(-, f)}{\longrightarrow}\left(-, A_{2}\right) \rightarrow F \rightarrow 0
$$

é exata. Então, tomando $C=\operatorname{coker}(f)$, e considerando, do item anterior, que $\left(-, A_{1}\right)$ e $\left(-, A_{2}\right)$ são projetivos, tem-se $F \cong(-, C)$. 


\section{Capítulo 2}

\section{A dimensão de representação}

\subsection{A motivação}

A dimensão de representação de uma álgebra de Artin é um valor associado a essa álgebra e foi introduzida com o objetivo de ajudar a entender o tipo de representação de uma álgebra, um dos grandes problemas na teoria de representações. Foi definido por Maurice Auslander e apresentado em uma apostila com título "Representation Dimension of Artin Algebras", publicado em 1971.. Por um longo período, esse conceito mantevese pouco utilizado, mas técnicas e pesquisas recentes vêm ajudando a desvendar melhor este número. Esta primeira seção destina-se a mostrar, de uma maneira não rigorosa, os resultados que Auslander apresenta como motivadores da definição de dimensão de representação. Estes resultados foram, posteriormente, melhor tratados em 1995, em [ARS95], quando são definidas as álgebras de Auslander. A partir desta seção, quando não mencionado, os símbolos $\Lambda$ e $\Gamma$ representam álgebras de Artin e os módulos serão módulos finitamente gerados sobre álgebras de Artin.

2.1 Definição. Para um módulo $M$, diz-se que $\operatorname{dom} \cdot \operatorname{dim}(M) \geqslant t$ quando dada uma resolução injetiva minimal de $M$

$$
0 \rightarrow M \rightarrow I_{0} \rightarrow I_{1} \rightarrow I_{2} \rightarrow \cdots \rightarrow I_{t} \rightarrow \cdots
$$

$I_{j}$ é projetivo para todo $j<t$.

O Teorema 2.4 abaixo fornece uma comparação entre as álgebras de tipo de repre- 
sentação finito e álgebras com restrições em suas dimensões global e dominante, as chamadas Álgebras de Auslander.

2.2 Definição. Uma álgebra de Artin $\Gamma$ é chamada álgebra de Auslander quando dom. $\operatorname{dim}(\Gamma) \geqslant 2$ e gl. $\operatorname{dim}(\Gamma) \leqslant 2$.

Observação 2.3. Observe que álgebras de Auslander admitem módulos finitamente gerados que são projetivos e injetivos simultaneamente. Portanto, considere $\Gamma$ uma álgebra de Auslander e seja $\mathcal{T}_{\Gamma}$ o subconjunto finito de objetos de ind $(\Gamma)$ formado pelos $\Gamma$-módulos projetivos-injetivos indecomponíveis. Denote por $\mathrm{Q}_{\Gamma}$ a soma $\sum_{\mathrm{Q} \in \mathcal{T}_{\Gamma}} \mathrm{Q}$.

O teorema abaixo, conforme comentado, compara álgebras de tipo de representação finito e álgebras de Auslander. Este teorema apresenta, na verdade, exemplos de álgebras de Auslander e sua prova pode ser encontrada por completo em [ARS95].

2.4 Teorema ([ARS95], cap. VI, 5.7). Seja $\mathbb{S}$ a coleção das classes de Morita-equivalência [ $\Lambda$ ] das álgebras de Artin de tipo finito $e \mathbb{V}$ a coleção das classes de Morita-equivalência [ $\Gamma]$ das álgebras de Auslander. Se ind $(\Lambda)=\left\{M_{i}\right\}_{i=1}^{n}$, e $\mathrm{Q}_{\Gamma}$ é o módulo segundo a observação anterior, então as aplicações abaixo são bijeções inversas entre si:

$$
\begin{aligned}
& \mathfrak{F}: \mathbb{S} \rightarrow \mathbb{V} \quad \mathfrak{G}: \mathbb{V} \rightarrow \quad \mathbb{S} \\
& {[\Lambda] \mapsto\left[\operatorname{End}_{\Lambda}\left(\sum M_{i}\right)^{\text {op }}\right] \quad[\Gamma] \mapsto\left[\operatorname{End}_{\Gamma}\left(Q_{\Gamma}\right)^{\text {op }}\right]}
\end{aligned}
$$

De uma maneira simplificada, o que este teorema diz é que se $\Lambda$ é álgebra de Artin de tipo finito, associada a ela há uma álgebra $\Gamma=\mathfrak{F}(\Lambda)$ que satisfaz:

1. $\operatorname{gl} . \operatorname{dim}(\Gamma) \leqslant 2$;

2. dom. $\operatorname{dim}(\Gamma) \geqslant 2 \mathrm{e}$

3. $\wedge$ é Morita-equivalente a $\operatorname{End}_{\Gamma}\left(Q_{\Gamma}\right)^{\text {op }}$

O terceiro ponto acima será generalizado mais a frente (lema 2.13) sob a mesma hipótese de dimensão dominante maior que 2, substituindo a soma de projetivos-injetivos por um módulo bem conhecido, a envolvente injetiva da álgebra $\Lambda$, como se pode observar na Proposição 2.6, que segue o lema abaixo. Este lema apresenta a relação entre a dimensão dominante de $\wedge$ e seus somandos. 
2.5 Lema. Seja $\Gamma$ uma álgebra de Artin. Então dom. $\operatorname{dim}_{\Gamma}(\Gamma) \geqslant 2$ se e somente se dom. $\operatorname{dim}_{\Gamma}(\mathrm{P}) \geqslant 2$ para cada projetivo $\mathrm{P}$ em $\bmod (\Gamma)$.

\section{Demonstração.}

$(\Longleftarrow)$ Basta observar que $\Gamma$ é um projetivo finitamente gerado.

$(\Longrightarrow)$ Seja $\mathrm{P}$ um projetivo indecomponível finitamente gerado e considere

$$
0 \rightarrow P \stackrel{f}{\rightarrow} I_{0}(P) \stackrel{f_{1}}{\rightarrow} I_{1}(P) \rightarrow \ldots
$$

uma resolução injetiva minimal de P. Como P é indecomponível, a inclusão natural $\mathrm{P} \stackrel{\mathfrak{i}}{\hookrightarrow} \Gamma \Gamma$ induz um morfismo $i_{1}: I_{0}(P) \rightarrow I_{0}(\Gamma)$ que faz comutar o diagrama abaixo, onde $I_{0}(\Gamma)$ é uma envolvente injetiva de $\Gamma$.

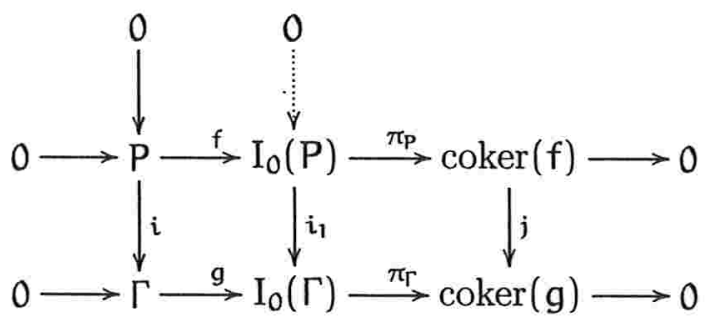

O morfismo $j$ é bem definido pela igualdade $j\left(p_{0}+i m(f)\right)=\pi_{\Gamma}\left(i_{1}\left(p_{0}\right)\right)$, e satisfaz $j \pi_{\mathrm{p}}=\pi_{\Gamma} i_{1}$.

A seta pontilhada indica que $i_{1}$ é monomorfismo: com efeito, sabendo que gi é monomorfismo (composta de monomorfismos) e que, portanto, gi $=i_{1} f$ é monomorfismo, conclui-se que $i_{1}$ é monomorfismo, uma vez que $f$ é monomorfismo essencial. E assim, por ser $I_{0}(P)$ um injetivo, $i_{1}$ cinde, e nesse caso $I_{0}(P)$ é somando de $I_{0}(\Gamma)$, que é projetivo. Portanto, $\mathrm{I}_{0}(\mathrm{P})$ também é projetivo.

Como os termos do meio nas seqüências acima são injetivos, o Lema $1.9 \mathrm{diz}$ que $I_{0}(\Gamma) \oplus \operatorname{coker}(f) \cong I_{0}(P) \oplus \operatorname{coker}(g)$. Nesse caso, as envolventes injetivas dessas somas também serão isomorfas, ou seja

$$
\mathrm{I}_{0}\left(\mathrm{I}_{0}(\Gamma) \oplus \operatorname{coker}(f)\right) \cong \mathrm{I}_{0}\left(\mathrm{I}_{0}(\mathrm{P}) \oplus \operatorname{coker}(g)\right)
$$

Entretanto

$$
\begin{aligned}
I_{0}\left(I_{0}(\Gamma) \oplus \operatorname{coker}(f)\right) & =I_{0}\left(I_{0}(\Gamma)\right) \oplus I_{0}(\operatorname{coker}(f)) \\
& =I_{0}(\Gamma) \oplus I_{0}(\operatorname{coker}(f))
\end{aligned}
$$




$$
\begin{aligned}
\mathrm{I}_{0}\left(\mathrm{I}_{0}(\mathrm{P}) \oplus \operatorname{coker}(\mathrm{g})\right) & =\mathrm{I}_{0}\left(\mathrm{I}_{0}(\mathrm{P})\right) \oplus \mathrm{I}_{0}(\operatorname{coker}(\mathrm{g})) \\
. & =\mathrm{I}_{0}(\mathrm{P}) \oplus \mathrm{I}_{0}(\operatorname{coker}(\mathrm{g})) .
\end{aligned}
$$

Então, da equação (2.1),

$$
\mathrm{I}_{0}(\Gamma) \oplus \mathrm{I}_{0}(\operatorname{coker}(f)) \cong \mathrm{I}_{0}(P) \oplus \mathrm{I}_{0}(\operatorname{coker}(g))
$$

$\mathrm{E}$ como $\mathrm{I}_{0}(\operatorname{coker}(f)) \cong \mathrm{I}_{1}(\mathrm{P})$ e $\mathrm{I}_{0}(\operatorname{coker}(\mathrm{g})) \cong \mathrm{I}_{1}(\Gamma)$, vale

$$
\mathrm{I}_{0}(\Gamma) \oplus \mathrm{I}_{1}(\mathrm{P}) \cong \mathrm{I}_{0}(\mathrm{P}) \oplus \mathrm{I}_{1}(\Gamma)
$$

Mas por hipótese, $I_{1}(\Gamma)$ é projetivo. Logo, $I_{1}(P)$ é somando de projetivo, portanto projetivo.

Seja $\mathrm{T}$ agora um projetivo finitamente gerado qualquer. Considere sua decomposição em indecomponíveis $T=\oplus \mathrm{P}_{j}$, com $\mathrm{P}_{j} \in$ ind $(\Gamma)$. Observe que cada $\mathrm{P}_{j}$ é projetivo indecomponível. Um argumento semelhante ao usado acima sobre a inclusão natural $P_{j} \hookrightarrow T$ mostra que se $0 \rightarrow \mathrm{T} \rightarrow \mathrm{I}_{0}(\mathrm{~T}) \rightarrow \mathrm{I}_{1}(\mathrm{~T}) \rightarrow \ldots$ é uma resolução injetiva minimal de $\mathrm{T}$, então $I_{0}(T)$ e $I_{1}(T)$ são projetivos.

2.6 Proposição. Se dom. $\operatorname{dim}(\Gamma) \geqslant 2$, então uma envolvente injetiva de $\Gamma$, digamos $\mathrm{I}_{0}(\Gamma)$, tem a propriedade que $\Lambda=\operatorname{End}\left(\mathrm{I}_{0}(\Gamma)\right)^{\text {op }}$ é Morita-equivalente a $\Lambda^{\prime}=\operatorname{End}\left(\mathrm{Q}_{\Gamma}\right)^{\mathrm{op}}$. Além disso, todo projetivo finitamente gerado está em $\mathrm{J}_{1}^{\mathrm{I}_{0}(\Gamma)}$.

Demonstração. Suponha que as categorias $\operatorname{add}\left(\mathrm{I}_{0}(\Gamma)\right)$ e $\operatorname{add}\left(\mathrm{Q}_{\Gamma}\right)$ sejam iguais.

Então as categorias $\mathcal{P}_{1}^{\mathrm{I}_{0}(\Gamma)}$ e $\mathcal{P}_{1}^{\mathrm{Q}_{\Gamma}}$ também serão iguais, bem como $\mathcal{J}_{1}^{\mathrm{I}_{0}(\Gamma)}$ e $\mathcal{J}_{1}^{\mathrm{Q}_{\Gamma}}$.

O Lema 1.10 associado ao Teorema 2.4 asseguram que

$$
\bmod (\Lambda) \simeq \mathcal{P}_{1}^{\mathrm{I}_{\mathrm{O}}(\Gamma)}=\mathcal{P}_{1}^{\mathrm{Q}_{\Gamma}} \simeq \bmod \left(\Lambda^{\prime}\right)
$$

desde que $I_{0}(\Gamma)$ seja projetivo, o que é verdadeiro por hipótese.

Então basta verificar que $\operatorname{add}\left(\mathrm{I}_{0}(\Gamma)\right)=\operatorname{add}\left(\mathrm{Q}_{\Gamma}\right)$.

Seja $M \in \operatorname{add}\left(I_{0}(\Gamma)\right)$. Então $M$ é soma direta de somandos indecomponíveis de $I_{0}(\Gamma)$. Observe que os somandos indecomponíveis de $\mathrm{I}_{0}(\Gamma)$ são isomorfos a elementos de $\mathcal{T}_{\Gamma}$, pois $I_{0}(\Gamma)$ é projetivo-injetivo finitamente gerado. Portanto $M$ é soma direta de $\Gamma$-módulos indecomponíveis em $\operatorname{add}\left(\mathrm{Q}_{\Gamma}\right)$. 
Por outro lado, seja $Q_{j} \in \mathcal{T}_{\Gamma}$. Como $Q_{j}$ é projetivo indecomponível, considere a inclusão natural $Q_{j} \stackrel{i}{\hookrightarrow} \Gamma$. Assim, compondo $i$ com uma envolvente injetiva de $\Gamma$, digamos $\Gamma \stackrel{e}{\hookrightarrow} \mathrm{I}_{0}(\Gamma)$, tem-se a seqüência exata $0 \rightarrow Q_{j} \stackrel{\text { ei }}{\rightarrow} \mathrm{I}_{0}(\Gamma)$ que cinde, já que $Q_{j}$ é injetivo. Logo, $Q_{j}$ é somando de $I_{0}(\Gamma)$ e portanto $Q_{j} \in \operatorname{add}\left(I_{0}(\Gamma)\right)$, para cada $Q_{j} \in \mathcal{T}_{\Gamma}$. Portanto, $\operatorname{add}\left(Q_{\Gamma}\right)=\operatorname{add}\left(I_{0}(\Gamma)\right)$.

Para observar que todo projetivo $P$ está em $\mathcal{J}_{1}^{\mathrm{I}_{0}(\Gamma)}=\mathcal{J}_{1}^{\mathrm{Q}_{\Gamma}}$, considere uma resolução injetiva minimal de $\mathrm{P}$, digamos

$$
0 \rightarrow \mathrm{P} \rightarrow \mathrm{I}_{0}(\mathrm{P}) \rightarrow \mathrm{I}_{1}(\mathrm{P}) \rightarrow \mathrm{I}_{2}(\mathrm{P}) \rightarrow \ldots
$$

Do Lema 2.5, $\mathrm{I}_{0}(\mathrm{P})$ e $\mathrm{I}_{1}(\mathrm{P})$ são projetivos. Portanto, são projetivos-injetivos, e estão $\mathrm{em} \operatorname{add}\left(\mathrm{Q}_{\Gamma}\right)=\operatorname{add}\left(\mathrm{I}_{0}(\Gamma)\right)$.

Então,

$$
0 \rightarrow \mathrm{P} \rightarrow \mathrm{I}_{0}(\mathrm{P}) \rightarrow \mathrm{I}_{1}(\mathrm{P})
$$

é uma $\operatorname{add}\left(\mathrm{I}_{0}(\Gamma)\right)$-apresentação deP, e assim, $\mathrm{P} \in \mathrm{J}_{1}^{\mathrm{I}_{0}(\Gamma)}$.

\subsection{A definição}

Com os resultados da primeira seção em mãos, pode-se agora apresentar a definição dada por Auslander para a dimensão de representação. O título desta seção, a rigor, deveria ser "As definições", porque há um esforço em caracterizar a dimensão de representação em termos de geradores e cogeradores. 'Tal caracterização já havia sido dada por Auslander naquele primeiro trabalho sobre essa dimensão, mas usando outra nomenclatura. Os trabalhos atuais tendem a usar como definição essa caracterização (e eventualmente a outra, dada na próxima seção), e o objetivo desta seção é apresentar o elo de ligação (Teorema 2.11 e Corolário 2.16) entre as duas definições que apareccm na literatura.

2.7 Definição. [Aus71]

A Dimensão de Representação de uma álgebra de Artin $\Lambda$ é o número

$$
\text { rep. } \operatorname{dim}(\Lambda)=\inf _{\Gamma \in A(\Lambda)}\{\text { gl. } \operatorname{dim}(\Gamma)\}
$$

onde $A(\mathcal{\Lambda})$ é a classe das álgebras de Artin que satisfazem: 
- dom. $\operatorname{dim}(\Gamma) \geqslant 2 \mathrm{e}$

- $\wedge$ é Morita-equivalente a $\operatorname{End}_{\Gamma}\left(I_{0}(\Gamma)\right)^{\text {op }}$.

A princípio, $A(\Lambda)$ poderia ser vazia. O resultado em 2.11.b mostra que isso não ocorre. Para isso, é necessário definir módulos geradores e cogeradores, com os quais se construirão as álgebras que estarão em $A(\Lambda)$. Depois de alguns lemas técnicos, dar-se-á uma resposta completa para a questão levantada acima.

2.8 Definição. Um $\Lambda$-módulo $X$ é dito gerador de $\bmod (\Lambda)$ quando para todo módulo $N \in \bmod (\Lambda)$, existe um epimorfismo $f: X^{\prime} \rightarrow N$ com $X^{\prime} \in \operatorname{add}(X)$.

Um $\Lambda$-módulo $Y$ é dito cogerador de $\bmod (\Lambda)$ quando para todo módulo $N \in \bmod (\Lambda)$, existe um monomorfismo $f: N \rightarrow Y^{\prime} \operatorname{com} Y^{\prime} \in \operatorname{add}(Y)$.

Observação 2.9. Sempre existem geradores e cogeradores para qualquer álgebra de Artin. De fato, o item (iii) da proposição abaixo mostra que $X=\oplus M_{i}$ é gerador de $\bmod (\Lambda)$, onde $\left\{M_{i}\right\}$ é um conjunto contendo todos os $\Lambda$-módulos projetivos de ind $(\Lambda)$. Analogamente, se $\left\{N_{j}\right\}$ é um conjunto contendo todos os $\Lambda$-módulos injetivos indecomponíveis dois a dois não-isomorfos, então $Y=\oplus N_{j}$ é um cogerador de $\bmod (\Lambda)$.

Em particular, se $\Lambda$ é álgebra de tipo finito, o módulo $\bigoplus M$ é um gerador-cogerador $\operatorname{de} \bmod (\Lambda)$ $M \in \operatorname{ind}(\Lambda)$

2.10 Proposição. As seguintes afirmações são equivalentes para um ^-módulo X:

i) X é gerador (resp. cogerador) de $\bmod (\Lambda)$;

ii) todo módulo projetivo (resp. injetivo) está em $\operatorname{add}(\mathrm{X})$;

iii) todo indecomponível projetivo (resp. injetivo) é somando de X.

Demonstração. A demonstração será feita para o caso "gerador". O caso "cogerador" tem prova dual.

i) $\Rightarrow$ ii) Seja $P$ um projetivo. Por ser $X$ um gerador de $\bmod (\Lambda)$, existe um epimorfismo $f: N \rightarrow P \operatorname{com} N \in \operatorname{add}(X)$. Logo, a seqüência exata abaixo cinde:

$$
0 \rightarrow \operatorname{ker}(\mathrm{f}) \rightarrow \mathrm{N} \stackrel{\mathrm{f}}{\rightarrow} \mathrm{P} \rightarrow 0,
$$


ou seja, $P \oplus \operatorname{ker}(f) \cong N$. Mas $N \in \operatorname{add}(X)$, que é categoria fechada para somandos e isomorfismos, de modo que $\mathrm{P} \in \operatorname{add}(\mathrm{X})$.

ii) $\Rightarrow$ iii) Se $P$ é projetivo indecomponível, por hipótese existem $n \in \mathbb{N}$ e $E \in \bmod (\Lambda)$ tais que $P \oplus E=n X$. Fazendo $X=\oplus_{i=1}^{r} J_{i}$ com $J_{i}$ indecomponível para $i \in\{1, \ldots, r\}$, o Teorema de Krull-Schmidt (1.1) nos assegura que existe $s \in\{1, \ldots, r\}$ tal que $\mathrm{P} \cong \mathrm{J}_{s}$, como queríamos.

iii) $\Rightarrow$ i) Seja $M \in \bmod (\Lambda)$, e considere $f: P_{0} \rightarrow M$ sua cobertura projetiva. O morfismo $f$ é epimorfismo, e $P_{0}$ é soma de projetivos indecomponíveis. Então, para cada $M \in \bmod (\Lambda)$, existe um epimorfismo $f: P_{0} \rightarrow M$ com $P_{0} \in \operatorname{add}(X)$. Assim, $X$ é gerador de $\bmod (\Lambda)$.

Com estas informações estabelecidas, é possível então enunciar o teorema mais importante desta seção. A primeira afirmação diz como são as álgebras em $A(\Lambda)$, enquanto a segunda resolve o problema da "não-vacuidade" de $A(\Lambda)$.

\subsection{Teorema.}

a) $S e \Gamma \in \mathrm{A}(\Lambda)$, então existe um $\Lambda$-módulo $\mathrm{M} \in \bmod (\Lambda)$ gerador-cogerador, tal que $\Gamma$ é Morita-equivalente a End ${ }_{\wedge}(M)^{\text {op }}$.

b) Reciprocamente, se $M \in \bmod (\Lambda)$ é gerador-cogerador, então $\Gamma=\operatorname{End}_{\Lambda}(M)^{\text {op }} \in A(\Lambda)$.

A demonstração deste teorema depende de alguns lemas técnicos, cujas demonstrações fazem uso intenso da linguagem categórica, e o Lema 2.12 necessita do uso dos funtores Ext. A abordagem de cálculo desses funtores segue de perto a usada por J. Rotman, na referência abaixo. A demonstração do Teorema 2.11 encontra-se na página 29 deste texto.

2.12 Lema. Seja $\Lambda$ uma álgebra de Artin e $M \in \bmod (\Lambda)$ um gerador de $\bmod (\Lambda)$.

1. Seja $\mathrm{C}_{1} \rightarrow \mathrm{C}_{2} \rightarrow \mathrm{C}_{3}$ uma sequência (não necessariamente exata) de $\Lambda$-módulos. Se $\left(-, C_{1}\right) \rightarrow\left(-, C_{2}\right) \rightarrow\left(-, C_{3}\right)$ é uma sequência exata em $\mathcal{F}_{M}$, então $C_{1} \rightarrow C_{2} \rightarrow C_{3}$ é uma sequência exata. 
2. Um ^-módulo $\mathrm{C}$ é injetivo se e somente se $(-, \mathrm{C})$ é injetivo em $\mathcal{F}_{\mathrm{M}}$.

3. Uma sequência exata $0 \rightarrow \mathrm{C} \rightarrow \mathrm{I}_{0} \rightarrow \mathrm{I}_{1}$ de $\Lambda$-módulos em add( $\left.\mathrm{M}\right)$ é uma coapresentação injetiva minimal de $\mathrm{C}$ se e somente se

$$
0 \rightarrow(-, C) \rightarrow\left(-, I_{0}\right) \rightarrow\left(-, I_{1}\right)
$$

é coapresentação injetiva minimal de $(-, \mathrm{C})$ em $\mathcal{F}_{\mathrm{M}}$.

Demonistração. 1. Seja $C_{1} \rightarrow C_{2} \rightarrow C_{3}$ nas condições acima. Então

$$
\left(\Lambda, C_{1}\right) \rightarrow\left(\Lambda, C_{2}\right) \rightarrow\left(\Lambda, C_{3}\right)
$$

é exata, já que $\Lambda \in \operatorname{add}(M)$, pois $M$ é gerador de $\bmod (\Lambda)$. Como $(\Lambda, C) \cong C$, é exata $\mathrm{C}_{1} \rightarrow \mathrm{C}_{2} \rightarrow \mathrm{C}_{3}$.

2. Seja $C \in \operatorname{add}(M)$. Então, conforme Rotman [Rot79, 7.12] $(-, C)$ é injetivo em $\mathcal{F}_{M}$ se, e somente se $\operatorname{Ext}^{1}(F,(-, C))=0$ para todo $F \in \mathcal{F}_{M}$. (Recorde que o funtor $(-, C)$ pode ser interpretado como módulo).

A prova consiste em mostrar que esta última afirmação é equivalente a dizer que $C$ é injetivo em $\bmod (\Lambda)$

Antes, observe que a categoria $\mathcal{F}_{M}$ tem dimensão global igual a dois, pois se $\mathrm{F} \in \mathcal{F}_{M}$, então existe um morfismo $f: A_{1} \rightarrow A_{2}$ tal que

$$
\left(-, A_{1}\right) \stackrel{(-, f)}{\longrightarrow}\left(-, A_{2}\right) \rightarrow F \rightarrow 0 \text {. }
$$

Considerando o kernel de $f$, tem-se a seqüência exata

$0 \rightarrow \operatorname{ker} f \rightarrow A_{1} \stackrel{f}{\rightarrow} A_{2} \quad$ que gera

$$
0 \rightarrow(-, \operatorname{ker} f) \rightarrow\left(-, A_{1}\right) \stackrel{(-, f)}{\longrightarrow}\left(-, A_{2}\right) \rightarrow F \rightarrow 0
$$

que é uma resolução projetiva minimal de $F$, já que $(-, X)$ é projetivo, para qualquer $X \in \operatorname{add}(M)$.

Como isso vale para todo $\mathrm{F} \in \mathcal{F}_{M}$, então gl. $\operatorname{dim}\left(\mathcal{F}_{M}\right) \leqslant 2$.

Logo, fixe uma resolução projetiva do funtor $F$

$$
0 \rightarrow\left(-, C_{2}\right) \rightarrow\left(-, C_{1}\right) \rightarrow\left(-, C_{0}\right) \rightarrow F \rightarrow 0
$$


Então os grupos $\operatorname{Ext}^{i}(F,(-, C))$ são calculados da seguinte maneira:

Aplicando o funtor $\operatorname{Hom}_{\mathcal{F}_{M}}(-,(-, C))$ ao complexo

$$
0 \rightarrow\left(-, C_{2}\right) \rightarrow\left(-, C_{1}\right) \rightarrow\left(-, C_{0}\right)
$$

tem-se

$$
0 \rightarrow \operatorname{Hom}_{\mathcal{F}_{M}}\left(\left(-, C_{0}\right),(-, C)\right) \rightarrow \operatorname{Hom}_{\mathcal{F}_{M}}\left(\left(-, C_{1}\right),(-, C)\right) \rightarrow \operatorname{Hom}_{\mathcal{F}_{M}}\left(\left(-, C_{2}\right),(-, C)\right) \rightarrow 0 .
$$

A Observação 1.18 permite estabelecer que, para cada $i, \operatorname{Hom}_{\mathcal{F}_{M}}\left(\left(-, C_{i}\right),(-, C)\right) \cong$ $\left(C_{i}, C\right)$, e portanto, é exata a seqüência

$$
0 \rightarrow\left(C_{0}, C\right) \rightarrow\left(C_{1}, C\right) \rightarrow\left(C_{2}, C\right) \rightarrow 0
$$

Logo, Ext ${ }^{i}(F,(-, C))=0$ para cada $i \in \mathbb{N}$ e para cada $F \in \mathcal{F}_{M}$ se, e somente se, dada uma seqüência exata

$$
0 \rightarrow \mathrm{C}_{0} \rightarrow \mathrm{C}_{1} \rightarrow \mathrm{C}_{2} \rightarrow 0
$$

a seqüência

$$
\left(C_{0}, C\right) \rightarrow\left(C_{1}, C\right) \rightarrow\left(C_{2}, C\right) \rightarrow 0
$$

é exata, se e somente se, $C$ é injetivo.

3. Decorre dos itens anteriores.

2.13 Lema. Se $M$ é um gerador-cogerador de $\bmod (\Lambda)$ e se $\operatorname{dom} \cdot \operatorname{dim}(\Gamma) \geqslant 2$ onde $\Gamma=\operatorname{End}_{\Lambda}(M)^{\mathrm{op}}$, então $\Lambda \simeq_{\mathrm{m}} \operatorname{End}_{\Gamma}\left(\mathrm{I}_{0}(\Gamma)\right)^{\mathrm{op}}$, onde $\mathrm{I}_{0}(\Gamma)$ é uma envolvente injetiva de $\Gamma$. Demonstração. Observe que a subcategoria aditiva gerada por $\mathrm{I}_{0}(\Gamma)$ é a subcategoria de $\bmod (\Gamma)$ que contém todos os $\Gamma$-módulos finitamente gerados que são projetivos e injetivos. Considerando a equivalência no Lema 1.23, esta subcategoria corresponde aos funtores de $\mathcal{F}_{M}$ que são injetivos e projetivos. Recorde que no lema anterior, um funtor projetivoinjetivo deve ter a forma $(-, I)$ onde $I \in \operatorname{add}(M)$ é injetivo em $\bmod (\Lambda)$.

Assim, se $\left\{I_{j}\right\}_{j=1}^{\text {n }}$ é um conjunto contendo um representante de cada isoclasse dos indecomponíveis injetivos de $\bmod (\Lambda)$, então o funtor $\left(-, \sum_{j=1}^{n} I_{j}\right)$ gera todos os funtores injetivos e projetivos. 
Dessas considerações, segue que $\operatorname{End}_{\Gamma}\left(I_{0}(\Gamma)\right)^{\text {op }}$ e $\operatorname{End}_{\mathcal{F}_{M}}\left(\left(-, \sum_{j=1}^{n} I_{j}\right)\right)^{\text {op }}$

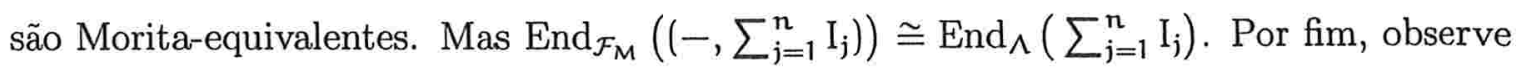
que $\operatorname{End}_{\Lambda}\left(\sum_{j=1}^{n} I_{j}\right)$ é Morita-equivalente a $\Lambda^{\text {op }}$. Daí, $\operatorname{End}_{\Gamma}\left(I_{0}(\Gamma)\right)^{\text {op }} \simeq_{m} \wedge$

2.14 Lema. Sejam $\Gamma$ uma álgebra de Artin e I um $\Gamma$-módulo injetivo. Então J I satisfaz:

1. Se $\mathrm{I}_{1}$ e $\mathrm{I}_{2}$ estão em $\mathrm{J}_{1}^{\mathrm{I}}$ e se $\mathrm{O} \rightarrow \mathrm{M} \rightarrow \mathrm{I}_{1} \rightarrow \mathrm{I}_{2}$ é exata, então $\mathrm{M} \in \mathrm{J}_{1}^{\mathrm{I}}$.

2. Um módulo $\mathrm{Q} \in \mathrm{J}_{1}^{\mathrm{I}}$ é injetivo nesta categoria se e somente se $\mathrm{Q} \in \operatorname{add}(\mathrm{I})$.

Demonstração. 1. É preciso encontrar uma add(I)-coapresentação para $M$.

Sejam $0 \rightarrow I_{1} \stackrel{d_{1}}{\longrightarrow} A_{1} \stackrel{d_{2}}{\longrightarrow} A_{2}$ e $0 \rightarrow I_{2} \stackrel{l_{1}}{\longrightarrow} B_{1} \stackrel{l_{2}}{\longrightarrow} B_{2}$ duas add(I)-coapresentações de $\mathrm{I}_{1}$ e $\mathrm{I}_{2}$, respectivamente.

Como $A_{2}$ e $B_{1}$ estão em add(I), $A_{2} \oplus B_{1}$ também está.

Considere o diagrama abaixo, onde $g_{1}$ é induzido por $g$.

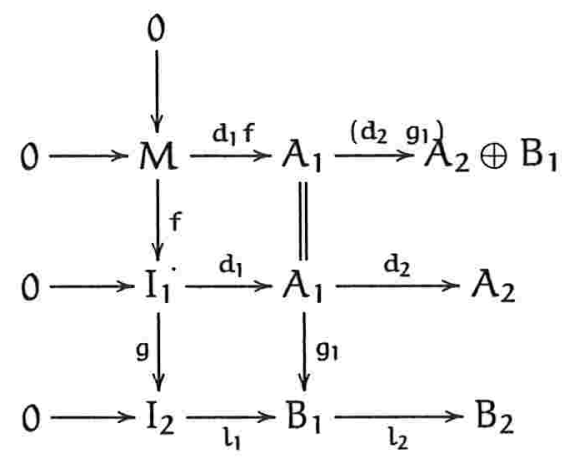

Se a sequência $O \rightarrow M \stackrel{d_{1} f}{\longrightarrow} A_{1} \stackrel{\left(d_{2} g_{1}\right)}{\longrightarrow} A_{2} \oplus B_{1}$ for exata, então tem-se o resultado.

Observe que $d_{1} f$ é monomorfismo, pois é composta de monomorfismos. Como $\left(\begin{array}{ll}d_{2} & g_{1}\end{array}\right) \circ d_{1} \circ f(m)=\left(d_{2} d_{1} f(m), g_{1} d_{1} f(m)\right)=\left(0, l_{1} g f(m)\right)=(0,0)$, tem-se $\operatorname{im}\left(d_{1} f\right) \subset \operatorname{ker}\left(d_{2} g_{1}\right)$.

Por outro lado, se $a \in \operatorname{ker}\left(d_{2} g_{1}\right)$, tem-se $d_{2}(a)=0$ e $g_{1}(a)=0$. Assim, $a \in \operatorname{ker}\left(d_{2}\right)=\operatorname{im}\left(d_{1}\right)$, ou seja, existe $i_{1} \in I_{1}$ tal que $d_{1}\left(i_{1}\right)=a$. Aplicando $g_{1}$ nessa igualdade, vem $0=g_{1}(a)=g_{1}\left(d_{1}\left(i_{1}\right)\right)=l_{1} g\left(i_{1}\right)$. Portanto $g\left(i_{1}\right) \in \operatorname{ker}\left(l_{1}\right)=\{0\}$, já que $l_{1}$ é monomorfismo.

Então $g\left(i_{1}\right)=0$ e $i_{1} \in \operatorname{ker}(g)=i m(f)$, o que implica que existe $m \in M$ tal que $f(m)=i_{1}$. Ou seja, existe $m \in M$ tal que $d_{1} f(m)=d_{1}\left(i_{1}\right)=a$. E assim, $a \in \operatorname{im}\left(d_{1} f\right)$. 


\section{2. $(\Rightarrow)$}

Existe uma sequência exata $0 \rightarrow \mathrm{Q} \rightarrow \mathrm{I}_{1} \rightarrow \mathrm{I}_{2}$, com $\mathrm{I}_{\mathrm{i}} \in \operatorname{add}(\mathrm{I})$. Como $\mathrm{Q}$ é injetivo em $\mathcal{J}_{1}^{\mathrm{I}}$, será somando de $\mathrm{I}_{1}$. Mas add(I) é fechada para somandos, e portanto $\mathrm{Q} \in \operatorname{add}(\mathrm{I})$.

$(\Leftarrow)$

Seja Q um módulo em add I. Q será injetivo em $J_{1}^{I}$ se, e somente se, dados o monomorfismo $0 \rightarrow M_{1} \stackrel{g}{\rightarrow} M_{2}$ e o morfismo $M_{1} \stackrel{f}{\rightarrow} Q$, na categoria $J_{1}^{I}$, exista um morfismo $M_{2} \stackrel{f^{\prime}}{\rightarrow} Q$ em $J_{1}^{I}$ tal que $f^{\prime} g=f$.

Sendo $\mathrm{Q}$ somando direto de um injetivo de $\bmod \Gamma$ (recorde que $\mathrm{Q} \in$ add $\mathrm{I})$, deverá ser injetivo em $\bmod \Gamma$. Neste caso, há um morfismo $f^{\prime}: M_{2} \rightarrow Q$ em $\bmod \Gamma$ de modo que vale $f^{\prime} g=f$. Como $J_{1}^{I}$ é subcategoria plena de $\bmod \Gamma$, então $f^{\prime}$ é morfismo de $\mathcal{J}_{1}^{\mathrm{I}}$, e portanto, o diagrama abaixo é comutativo em $\mathcal{J}_{1}^{\mathrm{I}}$.

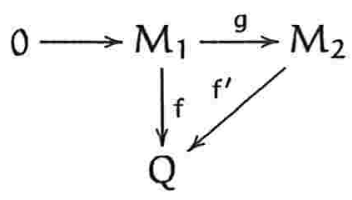

2.15 Lema. Se um $\Gamma$-módulo $\mathrm{P}$ em $\mathcal{J}_{1}^{\mathrm{I}_{0}(\Gamma)}$ é projetivo em $\mathrm{J}_{1}^{\mathrm{I}_{0}(\Gamma)}$ então $\mathrm{P}$ é $\Gamma$-módulo projetivo.

Demonstração. Se $\mathrm{P} \in \mathcal{J}_{1}^{\mathrm{I}_{0}(\Gamma)}$ é projetivo nesta categoria, e indecomponível, por ser $\Gamma$ uma álgebra de Artin, $\mathrm{P}$ admite uma cobertura projetiva $\mathrm{P}_{0} \stackrel{f}{\rightarrow} \mathrm{P} \rightarrow 0$. Como dom. $\operatorname{dim}(\Gamma) \geqslant 2$, o projetivo $P_{0}$ está em $J_{1}^{I_{0}(\Gamma)}$ (Proposição 2.6).

Neste caso, $\mathrm{f}$ é morfismo de $\mathcal{J}_{1}^{\mathrm{I}_{0}(\Gamma)}$, pois esta é uma subcategoria plena de $\bmod (\Gamma)$. Mais que isso, $f$ é epimorfismo em $\mathcal{J}_{1}^{\mathrm{I}_{0}(\Gamma)}$, uma vez que $f$ é epimorfismo em $\bmod (\Gamma)$.

Assim, sendo $f: P_{0} \rightarrow P$ um epimorfismo em $\mathcal{J}_{1}^{\mathrm{I}_{0}(\Gamma)}$ e sendo $P$ um projetivo nesta categoria, haverá um morfismo $g: P \rightarrow P_{0}$ de modo que $f g=I_{P}$. Diagramaticamente, significa que existe um morfismo $\mathrm{g}$ em $\mathcal{J}_{1}^{\mathrm{I}_{0}}(\Gamma)$ tal que, comuta o digrama abaixo.

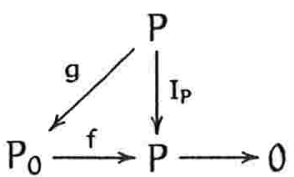


Como o morfismo g é morfismo em $\bmod (\Gamma)$, a seqüência exata $P_{0} \stackrel{f}{\rightarrow} P \rightarrow 0$ cinde, o que significa que $P$ é somando de $P_{0}$, que é projetivo em $\bmod (\Gamma)$. Logo, $P$ é projetivo em $\bmod (\Gamma)$.

\section{DemonstraÇÃo do TeOREMa 2.11.}

a) Esta demonstração será dividida em três partes. Primeiro, será construído um $\Lambda$ módulo $M$ finitamente gerado, que satisfaça $\Gamma \simeq_{m} \operatorname{End}_{\Lambda}(M)^{o p}$. Posteriormente, mostrarse-á que o módulo $M$ construído é gerador-cogerador.

Com efeito, se $\Gamma \in A(\Lambda)$, por definição, $\Lambda$ é Morita-equivalente a $\operatorname{End} \Gamma\left(I_{0}(\Gamma)\right)^{\text {op }}$, onde $0 \rightarrow \Gamma \stackrel{i}{\rightarrow} I_{0}(\Gamma)$ é uma envolvente injetiva de $\Gamma$.

Como $\mathrm{I}_{0}(\Gamma)$ é injetivo, o Lema 1.10 garante que $\mathcal{J}_{1}^{\mathrm{I}_{0}(\Gamma)}$ é categoria equivalente a $\bmod \left(\Lambda^{\prime}\right)$, onde $\Lambda^{\prime}=\operatorname{End}_{\Gamma}\left(\mathrm{I}_{0}(\Gamma)\right)^{\text {op }}$.

Neste caso, $\Lambda$ é Morita-equivalente a $\Lambda^{\prime}$, e portanto, $\mathcal{J}_{1}^{\mathrm{I}_{0}(\Gamma)}$ é equivalente a $\bmod (\Lambda)$.

Seja $\varphi: J_{1}^{I_{0}(\Gamma)} \rightarrow \bmod (\Lambda)$ uma particular equivalência.

Como dom. $\operatorname{dim}(\Gamma) \geqslant 2$, a Proposição 2.6 diz que todo projetivo de $\bmod (\Gamma)$ está na categoria $\mathcal{J}_{1}^{\mathrm{I}_{0}(\Gamma)}$. Então o $\Gamma$-módulo projetivo $\mathrm{Q}=\oplus \mathrm{P}_{\mathrm{i}}$ (onde $\left\{\mathrm{P}_{1}, \mathrm{P}_{2}, \ldots, \mathrm{P}_{\mathrm{t}}\right\}$ é um conjunto com todos os projetivos indecomponíveis não-isomorfos dois a dois) está em $\mathcal{J}_{1}^{\mathrm{I}_{0}}(\Gamma)$.

Seja $M=\varphi(Q) \in \bmod (\Lambda)$. Para este $\Lambda$-módulo $M$ vale $\Gamma \simeq_{m} \operatorname{End}_{\Lambda}(M)^{\text {op }}$.

De fato, como $\Gamma \in \operatorname{add}(Q)$, o Teorema de Morita (1.13) diz que $\Gamma \simeq_{m} \operatorname{End}_{\Gamma}(Q)^{o p}$. E já que $\varphi$ é equivalência, $\operatorname{End}_{\Gamma}(Q)^{o p} \cong \operatorname{End}_{\Lambda}(M)^{o p} . \operatorname{Então}^{\circ} \Gamma \simeq_{m} \operatorname{End}_{\Gamma}(Q)^{o p} \cong \operatorname{End}_{\Lambda}(M)^{o p}$.

O próximo passo é mostrar que $M$ é cogerador de $\bmod (\Lambda)$.

Para isso, recorde que a Proposição 2.10 exige de $M$ que todo injetivo indecomponível seja somando direto de $M$.

Seja, pois, $N$ um injetivo indecomponível em $\bmod (\Lambda)$.

Pela equivalência $\varphi$, considere $\mathrm{Q}^{\prime} \in \mathcal{J}_{1}^{\mathrm{I}_{0}(\Gamma)}$ tal que $\varphi\left(\mathrm{Q}^{\prime}\right)=\mathrm{N}$.

Observe que $\mathrm{Q}^{\prime}$ é indecomponível e injetivo na categoria $\mathcal{J}_{1}^{\mathrm{I}_{0}(\Gamma)} 1$

Se $Q^{\prime}$ for somando de $Q$, então $\varphi\left(Q^{\prime}\right)=N$ será somando de $\varphi(Q)=M$ e esta parte da demonstração termina.

Do Lema 2.14, $\mathrm{Q}^{\prime}$ ser injetivo em $\mathcal{J}_{1}^{\mathrm{I}_{0}(\Gamma)}$ implica que $\mathrm{Q}^{\prime} \in \operatorname{add}\left(\mathrm{I}_{0}(\Gamma)\right)$.

\footnotetext{
${ }^{1}$ Para definição de injetivos e projetivos em categorias, veja a página 16.
} 
Mas sendo $\mathrm{Q}^{\prime}$ indecomponível, deverá ser somando de $\mathrm{I}_{0}(\Gamma)$. Por ser este injetivoprojetivo, deverá $\mathrm{Q}^{\prime}$ ser injetivo-projetivo em $\bmod (\Gamma)$.

Como Q foi construído somando os indecomponíveis não-isomorfos (dois a dois) projetivos em $\bmod (\Gamma)$, então, $Q^{\prime}$ é um somando de $Q$. E sssim $N$ é somando de $M$, o que permite concluir que $M$ é um cogerador de $\bmod (\Lambda)$.

Por fim, basta verificar que $M$ é gerador de $\bmod (\Lambda)$.

Seja $N$ um projetivo indecomponível em $\bmod (\Lambda)$. Pela equivalência $\varphi$, considere $\mathrm{Q}^{\prime} \in \mathcal{J}_{1}^{\mathrm{I}_{0}(\Gamma)}$ tal que $\varphi\left(\mathrm{Q}^{\prime}\right)=\mathrm{N}$.

Observe que $\mathrm{Q}^{\prime}$ é indecomponível e projetivo em $\mathcal{J}_{1}^{\mathrm{I}_{0}(\Gamma)}$.

Se $Q^{\prime}$ for somando de $Q$, então $N$ será somando de $M$, e como no caso acima, pela Proposição $2.10, M$ será um gerador de $\bmod (\Lambda)$.

Do Lema 2.15, se $\mathrm{Q}^{\prime}$ é projetivo em $\mathcal{J}_{1}^{\mathrm{I}_{0}(\Gamma)}$ e indecomponível, então $\mathrm{Q}^{\prime}$ é projetivo em $\bmod (\Gamma)$ e indecomponível.

Recorde que Q foi construído somando projetivos indecomponíveis de $\bmod (\Gamma)$. Então, $\mathrm{Q}^{\prime}$ é um somando de $\mathrm{Q}$, e assim, $\mathrm{N}$ é somando de $\mathrm{M}$.

Logo, $M$ é um gerador de $\bmod (\Lambda)$, e com isso, fica provado que $M$ é um geradorcogerador de $\bmod (\Lambda)$.

b) Para que $\Gamma \in A(\Lambda)$, deve ser verificado que

i) $\operatorname{dom} \cdot \operatorname{dim}_{\Gamma}(\Gamma) \geqslant 2$

ii) $\Lambda \simeq_{m} \operatorname{End}_{\Gamma}\left(I_{0}(\Gamma)\right)^{o p}$

Sob a hipótese de dimensão dominante maior ou igual a 2, a parte (ii) acima é exatamente o que afirma o Lema 2.13.

E uma vez que se demonstre que todo módulo projetivo $X$ em $\bmod (\Gamma)$ tenha dimensão dominante no mínimo 2 , fica provado que dom. $\operatorname{dim}(\Gamma) \geqslant 2$, já que $\Gamma$ é $\Gamma$-módulo projetivo. Ou seja, estará completa a prova de (b).

Seja $X$ um $\Gamma$-módulo projetivo finitamente gerado. O Lema 1.23 diz que existe uma equivalência $\mathfrak{F}: \mathcal{F}_{M} \rightarrow \bmod (\Gamma)$. Considere $F=\mathfrak{F}^{-1}(X)$, onde $\mathfrak{F}^{-1}$ é uma equivalência inversa de $\mathfrak{F}$. Como $X$ é projetivo, o funtor $F$ será um funtor projetivo em $\mathcal{F}_{M}$. A 
Proposição 1.28 caracteriza os funtores projetivos em $\mathcal{F}_{M}$, garantindo a existência de um $\mathrm{C}$ em $\operatorname{add}(M)$ de modo que $F \cong(-, C)$.

Considere, para este $C$, uma coapresentação injetiva minimal

$$
0 \rightarrow \mathrm{C} \rightarrow \mathrm{I}_{0}(\mathrm{C}) \rightarrow \mathrm{I}_{1}(\mathrm{C})
$$

Como $M$ é cogerador, a Proposição 2.10 diz que $I_{0}(C)$ e $I_{1}(C)$ estão em add( $\left.M\right)$.

Então, do Lema 2.12,

$$
0 \rightarrow(-, C) \rightarrow\left(-, I_{0}(C)\right) \rightarrow\left(-, I_{1}(C)\right)
$$

é uma coapresentação injetiva minimal de $(-, C)$. E da já citada Proposição 1.28 , são projetivos em $\mathcal{F}_{M}$ os funtores $\left(-, \mathrm{I}_{0}(\mathrm{C})\right)$ e $\left(-, \mathrm{I}_{1}(\mathrm{C})\right)$.

Observe que aquela coapresentação injetiva minimal pode ser alongada a uma resolução injetiva minimal

$$
0 \rightarrow(-, C) \rightarrow\left(-, I_{0}(C)\right) \rightarrow\left(-, I_{1}(C)\right) \rightarrow F_{2} \rightarrow F_{3} \rightarrow \ldots
$$

Tomando a equivalência $\mathfrak{F}$ sobre esta seqüência, tem-se

$$
0 \rightarrow X \rightarrow \mathfrak{F}\left(\left(-, \mathrm{I}_{0}(\mathrm{C})\right)\right) \rightarrow \mathfrak{F}\left(\left(-, \mathrm{I}_{1}(\mathrm{C})\right)\right) \rightarrow \mathfrak{F}\left(\mathrm{F}_{2}\right) \rightarrow \mathfrak{F}\left(\mathrm{F}_{3}\right) \rightarrow \ldots
$$

uma resolução injetiva minimal de $X$, em que os dois primeiros injetivos, $\mathfrak{F}\left(-, I_{0}(C)\right)$ e $\mathfrak{F}\left(-, \mathrm{I}_{1}(\mathrm{C})\right)$ são também projetivos, já que $\left(-, \mathrm{I}_{0}(\mathrm{C})\right)$ e $\left(-, \mathrm{I}_{1}(\mathrm{C})\right)$ são projetivos. Logo, dom. $\operatorname{dim}(X) \geqslant 2$, para cada $X \in \bmod (\Lambda)$ projetivo.

O próximo corolário, decorrente do Teorema 2.11, é freqüentemente usado como definição para a dimensão de representação. Esta caracterização será útil quando for necessário calcular a dimensão de representação de algumas classes de álgebras.

2.16 Corolário. Para uma álgebra de Artin $\wedge$ vale:

$$
\text { rep. } \operatorname{dim}(\Lambda)=\inf _{\Gamma \in A(\Lambda)}\{g l \cdot \operatorname{dim}(\Gamma)\}=
$$

$\inf \left\{\operatorname{gl} \cdot \operatorname{dim}\left(\operatorname{End}_{\wedge} M\right)^{\mathrm{op}}:\right.$ Mé gerador-cogerador de $\left.\bmod (\Lambda)\right\}$ 


\subsection{Uma outra caracterização}

Esta seção apresenta uma maneira de calcular a dimensão de representação, que está implícita no principal texto de M. Auslander sobre o assunto ([Aus71]) e em artigos mais recentes, como em [Dug02], [Guo05], [APT] e [Xi00]. A abordagem abaixo foi sintetizada por Coelho e Platzeck em [CP04], e mostra o uso de resoluções de aproximações no cálculo da dimensão de representação. Usando as idéias desta seção, serão apresentados exemplos no próximo capítulo.

Seja $\mathcal{C}$ uma subcategoria plena de $\bmod (\Lambda)$ e considere $C \stackrel{f}{\rightarrow} M$ um morfismo em $\Lambda$.

2.17 Definição. O morfismo f é uma $\mathcal{C}$-aproximação à esquerda para $M$ quando $\mathrm{C} \in \mathrm{C}$ e, para cada $X \in \mathcal{C}$, a seqüência abaixo é exata em $\mathbf{A b}$ :

$$
(X, C) \stackrel{(X, f)}{\longrightarrow}(X, M) \rightarrow 0
$$

Uma seqüência exata

$$
0 \rightarrow C_{r} \stackrel{f_{r}}{\rightarrow} \cdots \stackrel{f_{2}}{\longrightarrow} C_{1} \stackrel{f_{1}}{\longrightarrow} M \rightarrow 0
$$

é uma resolução de $\mathcal{C}$-aproximações de $\mathrm{M}$ quando $\mathrm{C}_{\mathbf{i}} \in \mathcal{C}$ e para cada $\mathrm{X} \in \mathcal{C}$, é exata a seqüência em $\mathrm{Ab}$

$$
0 \rightarrow\left(X, C_{r}\right) \stackrel{\left(X, f_{r}\right)}{\longrightarrow} \cdots \stackrel{\left(X, f_{1}\right)}{\longrightarrow}(X, M) \rightarrow 0 .
$$

O número r é chamado comprimento da resolução.

2.18 Exemplo. Se $Y \in \operatorname{add}(M)$, então um isomorfismo $f: Y \rightarrow Y$ é uma resolução de $\operatorname{add}(M)$-aproximações de comprimento 1 , pois $0 \rightarrow(X, Y) \stackrel{(X, f)}{\longrightarrow}(X, Y) \rightarrow 0$ é exata em Ab para qualquer $X \in \operatorname{add}(M)$.

2.19 Definição. Um $\Lambda$-módulo $M$ é chamado $r$-aproximador quando todo $\Lambda$-módulo admite uma resolução de $\operatorname{add}(M)$-aproximações de comprimento no máximo $r$.

O próximo lema é necessário para a caracterização dada à frente.

2.20 Lema. Seja $M \in \bmod (\Lambda)$

a) $S e \mathrm{Y} \in \bmod (\Lambda)$ tem uma resolução de $\operatorname{add}(\mathrm{M})$-aproximações com comprimento $\mathrm{s}$, então $\operatorname{pd}_{\mathcal{F}_{M}}(-, Y) \leqslant s-1$ 
b) Reciprocamente, se $\operatorname{pd}_{\mathcal{F}_{M}}(-, \mathrm{Y}) \leqslant \mathrm{s}-1$ e $\mathrm{M}$ é gerador de $\bmod (\Lambda)$, então $\mathrm{Y}$ tem uma resolução de $\operatorname{add}(\mathrm{M})$-aproximações de comprimento $\mathrm{s}$.

Demonstração.

a) Considere que a seqüência abaixo seja uma resolução de add( $M)$-aproximações de Y com comprimento s:

$$
0 \rightarrow M_{s-1} \rightarrow \ldots \rightarrow M_{0} \rightarrow Y \rightarrow 0
$$

Então, $M_{i} \in \operatorname{add}(M)$, para cada $i \in\{0, \ldots, s-1\}$. Por definição é exata em $\mathcal{F}_{M}$ a seqüência

$$
0 \rightarrow\left(-, M_{s-1}\right) \rightarrow \ldots \rightarrow\left(-, M_{0}\right) \rightarrow(-, Y) \rightarrow 0
$$

Como em $\mathcal{F}_{M}$ os projetivos são da forma $(-, C)$, com $C \in \operatorname{add}(M)$ (Proposição 1.28), são projetivos em $\mathcal{F}_{M}$ os funtores $\left(-, M_{i}\right)$.

Logo a seqüência (2.2) é uma resolução projetiva de $(-, Y)$ e portanto $\operatorname{pd}_{\mathcal{F}_{M}}(-, Y) \leqslant s-1$.

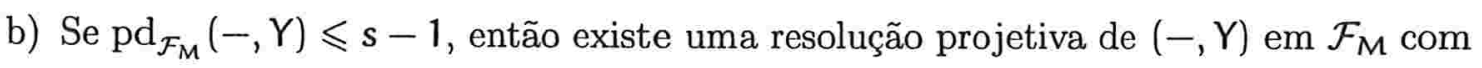
comprimento $s-1$. Novamente da Proposição 1.28, tem-se que os projetivos de $\mathcal{F}_{M}$ são da forma $(-, C) \operatorname{com} C \in \operatorname{add}(M)$. Logo uma resolução projetiva de $(-, Y)$ tem a forma

$$
0 \rightarrow\left(-, M_{s-1}\right) \stackrel{\varphi_{s-1}}{\longrightarrow} \ldots \stackrel{\varphi_{1}}{\longrightarrow}\left(-, M_{0}\right) \stackrel{\varphi_{0}}{\longrightarrow}(-, Y) \rightarrow 0
$$

$\operatorname{com} M_{\mathbf{i}} \in \operatorname{add}(M)$.

Como $M$ é gerador, a Proposição $2.10 \mathrm{diz}$ que $\Lambda \in \operatorname{add}(M)$. Então é exata a seqüência

$$
0 \rightarrow\left(\Lambda, M_{s-1}\right) \stackrel{\left[\varphi_{s-1}\right]_{\Lambda}}{\longrightarrow} \ldots \stackrel{\left[\varphi_{1}\right]_{\Lambda}}{\longrightarrow}\left(\Lambda, M_{0}\right) \stackrel{\left[\varphi_{0}\right]_{\Lambda}}{\longrightarrow}(\Lambda, Y) \rightarrow 0
$$

Cada morfismo entre colchetes é o morfismo pertencente à aplicação natural $\varphi_{i}$ da seqüência (2.3) correspondente a $\Lambda$. 
Mas $\operatorname{Hom}_{\Lambda}\left(\Lambda, M_{i}\right) \cong M_{i}$ mediante um isomorfismo $\theta_{i}$. Logo, é exata a seqüência induzida

$$
0 \rightarrow M_{s-1} \stackrel{f_{s-1}}{\longrightarrow} \ldots \stackrel{f_{1}}{\rightarrow} M_{0} \stackrel{f_{0}}{\longrightarrow} Y \rightarrow 0
$$

onde $f_{i}=\theta_{i-1}^{-1} \circ\left[\varphi_{i}\right]_{\wedge} \circ \theta_{i}$.

O lema estará provado se (2.5) for uma resolução de $\operatorname{add}(M)$-aproximações. Mas isso é verdade, uma vez que $M_{i} \in \operatorname{add}(M)$ e comuta o diagrama

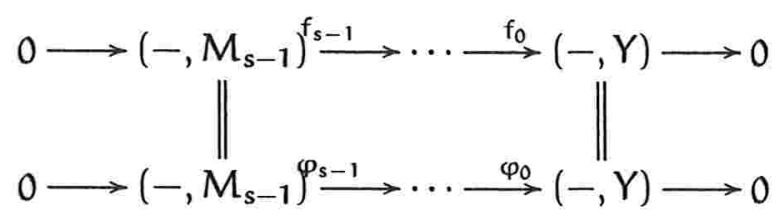

2.21 Teorema. Seja $\wedge$ uma álgebra de Artin. Se $\mathrm{r} \in \mathbb{N}$, são equivalentes:

1. $\operatorname{rep} \cdot \operatorname{dim}(\Lambda) \leqslant r+1$;

2. existe um gerador-cogerador de $\bmod (\Lambda)$ que é r-aproximador.

\section{Demonstração.}

$(1 \Rightarrow 2)$ Suponha que $s=\operatorname{rep} \operatorname{dim}(\Lambda) \leqslant r+1$

Do Corolário 2.16, existe um módulo gerador-cogerador $M \in \bmod (\Lambda)$ tal que

$$
\text { gl. } \operatorname{dim}(\Gamma)=s \text {, onde } \Gamma=\operatorname{End}(M)^{\text {op }} \text {. }
$$

Esta parte do teorema estará provada se $M$ for um r-aproximador.

Para tanto, é preciso verificar que todo $\Lambda$-módulo $X$ tem uma resolução de add( $M)$ aproximações com comprimento $r$.

Se $X \in \operatorname{add}(M)$, então considere um isomorfismo $f: X \rightarrow X$. Assim, a seqüência abaixo é uma resolução de $\operatorname{add}(M)$-aproximações com comprimento $r$ :

$$
0 \rightarrow C_{r} \rightarrow \ldots \rightarrow C_{2} \rightarrow C_{1} \stackrel{f}{\rightarrow} X \rightarrow 0
$$

onde $C_{1}=X$ e, para cada $j \neq 1, C_{j}=0$. 
Se $X \notin \operatorname{add}(M)$, considere uma coapresentação injetiva minimal de $X$, por exemplo $0 \rightarrow X \rightarrow I_{0} \stackrel{f_{1}}{\rightarrow} I_{1}$. Então o funtor $F=\left(-, \operatorname{coker}\left(f_{1}\right)\right)$ definido no Exemplo 1.22 é coerente, uma vez que $I_{0}$ e $I_{1}$ estão em $\operatorname{add}(M)$, pois $M$ é cogerador. Como no Exemplo citado, é exata a seqüência

$$
0 \rightarrow(-, X) \rightarrow\left(-, I_{0}\right) \stackrel{\left(-, f_{1}\right)}{\longrightarrow}\left(-, I_{1}\right) \rightarrow F \rightarrow 0
$$

Recorde que $\mathcal{F}_{M}$ e $\bmod (\Gamma)$ são categorias equivalentes (Lema 1.23), e assim

$$
\text { gl. } \operatorname{dim}\left(\mathcal{F}_{M}\right)=\text { gl. } \operatorname{dim}(\Gamma)
$$

Portanto $\operatorname{pd}_{\mathcal{F}_{M}}(F) \leqslant$ gl. $\operatorname{dim}\left(\mathcal{F}_{M}\right)=$ gl. $\operatorname{dim}(\Gamma)=s$.

Como $X \notin \operatorname{add}(M)$, então $(-, X)$ não é projetivo em $\mathcal{F}_{M}$ (Proposição 1.28).

Seja

$$
0 \rightarrow \mathrm{F}_{\mathrm{n}-1} \rightarrow \ldots \rightarrow \mathrm{F}_{0} \rightarrow(-, \mathrm{X}) \rightarrow 0
$$

uma resolução projetiva minimal de $(-, X)$, onde $n=\operatorname{pd}(-, X)$.

Então, colando as seqüências (2.6) e (2.7) tem-se a seqüência exata

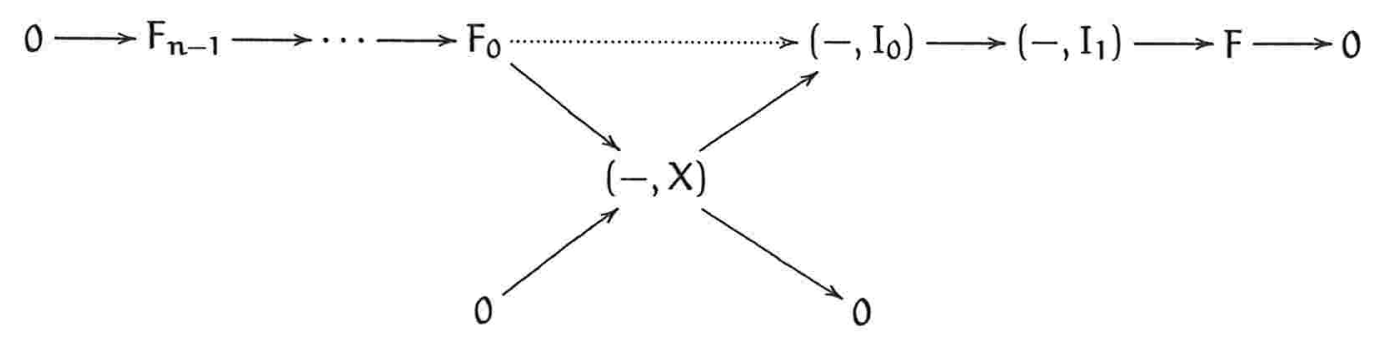

Assim, $\operatorname{pd}(F)=n+2 \leqslant g l \cdot \operatorname{dim}\left(\mathcal{F}_{M}\right)=s$. E daí $n+2 \leqslant s$, donde $n \leqslant s-2$, ou seja, $\operatorname{pd}(-, X) \leqslant s-2$.

Observe que o Lema anterior afirma que neste caso, $(-, X)$ admite uma resolução de $\operatorname{add}(M)$-aproximações com comprimento $s-1$. Seja

$$
0 \rightarrow \mathrm{F}_{s-1}^{\prime} \rightarrow \ldots \rightarrow \mathrm{F}_{1}^{\prime} \rightarrow \mathrm{F} \rightarrow 0
$$

uma tal resolução. Alongando esta resolução com "zeros", encontra-se uma resolução de $\operatorname{add}(M)$-aproximações com comprimento $r$, o que prova, portanto, que $M$ é $r$ aproximador. 
$(2 \Rightarrow 1)$ Suponha que $M$ seja um gerador-cogerador de $\bmod (\Lambda)$ que seja $r$-aproximador. Para demonstrar que rep. $\operatorname{dim}(\Lambda) \leqslant r+1$, basta mostrar que

$$
\text { gl. } \operatorname{dim}(\Gamma) \leqslant r+1, \text { onde } \Gamma=\operatorname{End}(M)^{\text {op }} .
$$

Mas, pela equivalência no Lema 1.23 , é suficiente mostrar que gl. $\operatorname{dim}\left(\mathcal{F}_{M}\right) \leqslant r+1$.

Seja $F$ um funtor coerente qualquer em $\mathcal{F}_{M}$. Por definição, existe um morfismo $f: X^{\prime \prime} \rightarrow X^{\prime}$ em add $(M)$ tal que

$$
\left(-, X^{\prime \prime}\right) \stackrel{(-, f)}{\longrightarrow}\left(-, X^{\prime}\right) \rightarrow F \rightarrow 0 \quad \text { é exata em } \mathcal{F}_{M}
$$

Seja $X=\operatorname{ker}(f)$. Então é exata a seqüência

$$
0 \rightarrow X \rightarrow X^{\prime \prime} \stackrel{f}{\rightarrow} X^{\prime}
$$

Como $M$ é r-aproximador, existe uma resolução de $\operatorname{add}(M)$-aproximações para $X$, com comprimento $r$.

Seja

$$
0 \rightarrow M_{r} \rightarrow \ldots \rightarrow M_{1} \stackrel{d_{1}}{\rightarrow} X \rightarrow 0
$$

uma tal resolução. Então, $M_{i} \in \operatorname{add}(M)$ para todo $i \in\{1, \ldots, r\}$ e

$$
0 \rightarrow\left(-, M_{r}\right) \rightarrow \ldots \rightarrow\left(-, M_{1}\right) \stackrel{\left(-, d_{1}\right)}{\longrightarrow}(-, X) \rightarrow 0 \quad \text { é exata em } \mathcal{F}_{M} .
$$

Como $X$ é kernel de $f$,

$$
0 \rightarrow(-, X) \rightarrow\left(-, X^{\prime \prime}\right) \stackrel{(-, f)}{\longrightarrow}\left(-, X^{\prime}\right) \rightarrow F \rightarrow 0 \quad \text { é exata em } \mathcal{F}_{M} .
$$

Colando (2.8) e (2.9) tem-se a seqüência exata $\delta$ em $\mathcal{F}_{M}$

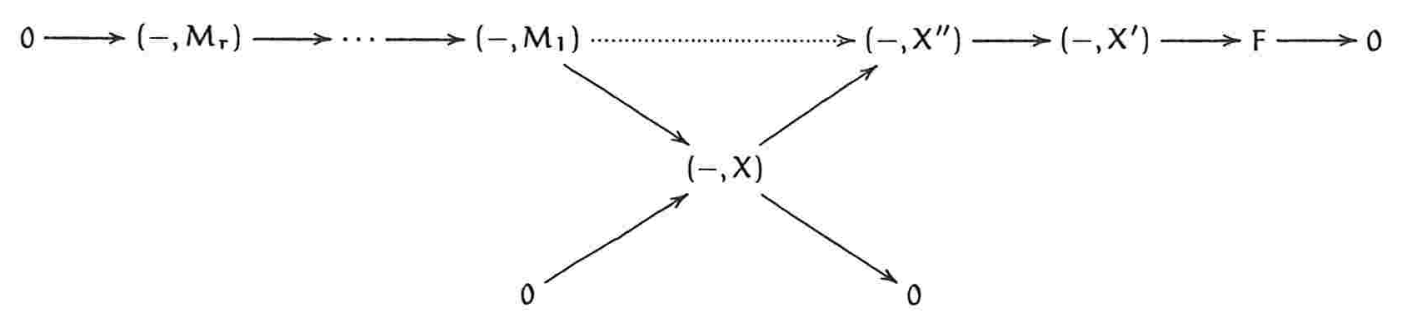

Recorde que $\left(-, M_{i}\right),\left(-, X^{\prime}\right)$ e $\left(-, X^{\prime \prime}\right)$ são projetivos em $\mathcal{F}_{M}$, e portanto $\delta$ é resolução projetiva de $F$. Então, $\operatorname{pd}_{\mathcal{F}_{M}}(F)=r+1$ para qualquer $F \in \mathcal{F}_{M}$. Neste caso, gl. $\operatorname{dim}\left(\mathcal{F}_{M}\right) \leqslant r+2$. E portanto, gl. $\operatorname{dim}(\Gamma) \leqslant r+2$. 
2.22 Corolário. Para uma álgebra de Artin $\Lambda$, vale:

rep. $\operatorname{dim}(\Lambda)=r+1 \Leftrightarrow$ existe um gerador-cogerador $r$-aproximador, mas não há nenhum gerador-cogerador s-aproximador, para $\mathrm{s}<\mathrm{r}$.

Demonstração. Com efeito, se houvesse um s-aproximador com $\mathrm{s}<\mathrm{r}$, então

$$
\text { rep. } \operatorname{dim}(\Lambda) \leqslant s+1<r+1=\text { rep. } \operatorname{dim}(\Lambda)
$$

e assim, $r=s$, contradição.

Por outro lado, se há um r-aproximador, do Teorema acima, rep. $\operatorname{dim}(\Lambda) \leqslant r+1$. Se, por absurdo, rep. $\operatorname{dim}(\Lambda)<r+1$, então $\operatorname{rep} \cdot \operatorname{dim}(\Lambda) \leqslant r$ e portanto haveria um $(r-1)$-aproximador, contradição. 


\section{Capítulo 3}

\section{Alguns Exemplos}

Neste capítulo pretende-se apresentar alguns exemplos de álgebras para as quais é possível encontrar a dimensão de representação usando as técnicas do capítulo anterior. No fim do capítulo são apresentados também resumos dos principais resultados sobre a dimensão de representação que se podem encontrar publicados.

A situação mais simples é aquela em que a álgebra é semi-simples. Para tais álgebras vale o seguinte:

3.1 Teorema. Seja $\wedge$ uma álgebra de Artin.

A álgebra $\wedge$ é semi-simples se, e somente se rep. $\operatorname{dim}(\Lambda)=0$.

\section{Demonstração.}

$(\Rightarrow)$ Se $\wedge$ é semi-simples, será semi-simples o módulo $D(\Lambda)$. Então é semi-simples a álgebra $\Gamma=$ End $(\Lambda \oplus D(\Lambda))^{\text {op }}$. Neste caso, rep. $\operatorname{dim}(\Lambda) \leqslant$ gl. $\operatorname{dim}(\Gamma)=0$, já que $\Lambda \oplus \mathrm{D}(\Lambda)$ é gerador-cogerador de $\Lambda$.

$(\Leftarrow)$ Se rep. $\operatorname{dim}(\Lambda)=0$, seja $M$ um gerador-cogerador de $\bmod (\Lambda)$ tal que

$$
0=\operatorname{rep} \cdot \operatorname{dim}(\Lambda)=\text { gl. } \operatorname{dim}\left(\operatorname{End}_{\Lambda}(M)\right)^{\text {op }}
$$

Se $\wedge$ não é semi-simples, então existe um projetivo indecomponível $\mathrm{P}$ com comprimento maior ou igual a 2. Como $M$ é gerador, $P$ é somando direto de $M$, e portanto $\operatorname{End}_{\Lambda}(M)^{\text {op }}$ não pode ser semi-simples, contradição. 
Observação 3.2. Algo importante de se observar é que não há álgebras de Artin com dimensão de representação igual a 1. Isso decorre diretamente do Teorema 2.21, uma vez que não podem ocorrer resoluções de aproximação de comprimento 0.

A dimensão de representação caracteriza as álgebras de tipo de representação finito. O próximo teorema, mais especificamente a equivalência entre (a) e (b), seguindo as idéias da seção (2.1) é que motiva Auslander a afirmar que é esperado que a dimensão de representação forneça uma maneira razoável de medir quão distante uma álgebra de Artin está de ser de tipo de representação finito [Aus71, pg 134].

3.3 Teorema. Para uma álgebra de Artin $\Lambda$, são equivalentes:

a) $\operatorname{rep} \cdot \operatorname{dim}(\Lambda) \leqslant 2$.

b) $\Lambda$ é álgebra de tipo finito.

c) Existe $M \in \bmod (\Lambda)$ tal que $\operatorname{add}(M)=\bmod (\Lambda)$.

d) Existe $M$ gerador-cogerador de $\bmod (\Lambda)$, 1-aproximador.

\section{Demonstração.}

$(\mathrm{a} \Rightarrow \mathrm{b})$ Para que $\wedge$ seja álgebra de tipo de representação finito, é necessário que haja um número finito de $\Lambda$-módulos indecomponíveis, finitamente gerados, dois a dois não isomorfos.

Seja $N$ um indecomponível de $\bmod (\Lambda)$.

Como rep. $\operatorname{dim}(\Lambda) \leqslant 2$, o Teorema 2.21 garante que existe um $\Lambda$-módulo $M$ geradorcogerador de $\bmod (\Lambda)$ que é 1 -aproximador.

Então, existe uma resolução de $\operatorname{add}(M)$-aproximações de $\mathrm{N}$ com comprimento 1. Seja $0 \rightarrow K \stackrel{f}{\rightarrow} N \rightarrow 0$ tal resolução, onde $K \in \operatorname{add}(M)$. Portanto, $K \cong N$ e daí $\mathrm{N} \in \operatorname{add}(\mathrm{M})$. Como $\mathrm{N}$ é indecomponível, deve ser um somando direto de $M$. Mas $M$ tem um número finito de somandos indecomponíveis (Teorema 1.1), de onde se conclui que existe apenas um número finito de indecomponíveis em $\bmod (\Lambda)$. 
$(b \Rightarrow c)$ Seja $M=\bigoplus_{M_{i} \in \operatorname{ind}(\Lambda)} M_{i}$. Então certamente $\bmod (\Lambda)=\operatorname{add}(M)$, uma vez que todo $\Lambda$-módulo finitamente gerado tem seus somandos diretos em $\operatorname{add}(M)$.

$(c \Rightarrow d)$ Se $M$ é tal que $\operatorname{add}(M)=\bmod (\Lambda)$, então $M$ é gerador-cogerador, uma vez que $\Lambda \in \operatorname{add}(M)$ e $D(\Lambda) \in \operatorname{add}(M)$ (Proposição 2.10). Este $M$ também é um 1-aproximador, pois, para cada $X \in \bmod (\Lambda)$, um isomorfismo $0 \rightarrow X \rightarrow X \rightarrow 0$ é uma resolução de $\operatorname{add}(M)$-aproximações de $X$ com comprimento 1.

$(d \Rightarrow a)$ Resulta imediatamente da implicação $(2 \Rightarrow 1)$ do Teorema 2.21 com $r=1$.

\subsection{Dimensão de Representação 3}

Na primeira parte deste capítulo observou-se caracterizações, por meio da dimensão de representação, das álgebras semi-simples e álgebras de tipo de representação finito. Nesta seção, as álgebras em questão são álgebras de tipo infinito.

Um dos fatos que pode ser motivador para encontrar álgebras com dimensão de representação no máximo 3 é aquele comentado na introdução desta dissertação, que é o resultado encontrado por Igusa e Todorov, em [IT02]. Segundo este artigo, quando uma álgebra de Artin $\Lambda$ tem dimensão de representação no máximo 3 , vale a conjectura finitística, ou seja, é finito o valor de

$$
\text { fin. } \operatorname{dim}(\Lambda)=\sup \left\{\operatorname{pd}_{\Lambda}(X): X \in \bmod (\Lambda) e \operatorname{pd}_{\Lambda}(X)<\infty\right\}
$$

A proposição abaixo decorre diretamente do Corolário 2.22 e da caracterização dada no Teorema 3.3 e será útil sob esta hipótese, para calcular a dimensão de representação de álgebras hereditárias, álgebras coladas a direita e álgebras laura.

3.4 Proposição. Seja $\wedge$ uma álgebra de Artin de tipo infinito. Então rep. $\operatorname{dim}(\Lambda)=3$ se e somente se existe um gerador-cogerador de $\bmod (\Lambda)$ que é 2 -aproximador.

\subsubsection{A dimensão de representação de álgebras hereditárias}

3.5 Definição. Uma álgebra de Artin $\Lambda$ é dita álgebra hereditária quando os submódulos do $\wedge$-módulo regular $\wedge \wedge$ são projetivos. 
Rotman ([Rot79, 4.23]) mostra que neste caso, se P é um $\Lambda$-módulo projetivo, então todo submódulo de $\mathrm{P}$ é projetivo. Além disso, se I é um $\Lambda$-módulo injetivo e I' é um submódulo de I, então o módulo quociente $\frac{\mathrm{I}}{\mathrm{I}^{\prime}}$ é injetivo.

Também é verdade que $\Lambda$ é hereditária se e somente se $\operatorname{gl} \operatorname{dim}(\Lambda) \leqslant 1$. Veja, por exemplo [ARS95, 5.2].

Exemplos de álgebras hereditárias são encontrados entre as álgebras de caminho cujos quívers não têm ciclos orientados (veja, por exemplo, [Coe92]).

O principal teorema desta seção é o que garante que a dimensão de representação de álgebras hereditárias de tipo infinito é igual a 3. Antes de provar este resultado, observe o seguinte lema:

3.6 Lema. Seja $\wedge$ uma álgebra hereditária. Se X é um ^-módulo indecomponível nãoinjetivo, então $\operatorname{Hom}_{\wedge}(\mathrm{I}, \mathrm{X})=0$, para qualquer injetivo I.

Demonstração. Seja $f \in \operatorname{Hom}_{\Lambda}(I, X)$ um morfismo qualquer.

Então é exata a seqüência abaixo:

$$
0 \rightarrow \operatorname{im}(f) \hookrightarrow X \rightarrow \frac{X}{\operatorname{im}(f)} \rightarrow 0 .
$$

Mas $\operatorname{im}(f) \cong \frac{I}{\operatorname{ker}(f)}$, pelo Teorema do homomorfismo (veja [AF92, 3.7]), e como $\wedge$ é hereditária, $\frac{\mathrm{I}}{\operatorname{ker}(f)}$ é injetivo. Portanto $\operatorname{im}(f)$ é injetivo e a seqüência acima cinde. Neste caso, $X=\operatorname{im}(f) \oplus T$, onde $T \cong \frac{X}{i m(f)}$. Mas $X$ é indecomponível, por hipótese. Então $T=0$ ou $\operatorname{im}(f)=0$. Se $T=0$, então $X$ é igual a $\operatorname{im}(f)$, que é um módulo injetivo, contrariando a hipótese. Assim, $\operatorname{im}(f)=0$, mostrando que $f$ é morfismo nulo. Logo, $\operatorname{Hom}_{\wedge}(I, X)=0$.

3.7 Teorema. Se $\wedge$ é uma álgebra hereditária de tipo de representação infinito, então rep. $\operatorname{dim}(\Lambda)=3$.

Demonstração. Segundo a Proposição 3.4, é suficiente encontrar um gerador-cogerador $\operatorname{de} \bmod (\Lambda)$ que seja 2-aproximador.

Considere $M=\Lambda \oplus D(\Lambda)$.

Como $\Lambda$ é soma direta de todos os projetivos indecomponíveis presentes em ind $(\Lambda)$ e $D(\Lambda)$ é soma direta de todos os injetivos indecomponíveis em ind $(\Lambda)$, então $M$ é geradorcogerador de $\bmod (\Lambda)$. 
Para mostrar que $M$ é também 2-aproximador, é necessário verificar que todo $\Lambda$ módulo $X$ admite uma resolução de $\operatorname{add}(M)$-aproximações com comprimento 2 .

Se $X \in \operatorname{add}(M), 0 \rightarrow X \stackrel{I_{X}}{\rightarrow} X \rightarrow 0$ é uma tal resolução.

Se $X \notin \operatorname{add}(M)$ e é indecomponível, considere uma resolução projetiva minimal de $X$, digamos

$$
\left.0 \rightarrow \mathrm{P}_{1} \rightarrow \mathrm{P}_{0} \stackrel{\mathrm{g}}{\rightarrow} \mathrm{X} \rightarrow 0 \text { (recorde que gl. } \operatorname{dim}(\Lambda) \leqslant 1\right)
$$

Como $P_{0}$ e $P_{1}$ são projetivos, estão em $\operatorname{add}(M)$. Para que esta resolução seja uma resolução de $\operatorname{add}(M)$-aproximações, basta que, para todo $Y \in \operatorname{add}(M)$, a seqüência de grupos abelianos

$$
0 \rightarrow\left(Y, P_{1}\right) \rightarrow\left(Y, P_{0}\right) \stackrel{(Y, g)}{\longrightarrow}(Y, X) \rightarrow 0 \text { seja exata. }
$$

Como

$$
0 \rightarrow\left(\mathrm{Y}, \mathrm{P}_{1}\right) \rightarrow\left(\mathrm{Y}, \mathrm{P}_{0}\right) \stackrel{(\mathrm{Y}, \mathrm{g})}{\longrightarrow}(\mathrm{Y}, \mathrm{X}) \text { sempre é exata, (Ver por exemplo [Ass97]). }
$$

Basta então mostrar que, para cada $Y \in \operatorname{add}(M),(Y, g)$ é sobrejetora.

Como $M=\Lambda \oplus D(\Lambda)$, se $Y \in \operatorname{add}(M)$, então os somandos diretos indecomponíveis de $Y$ são projetivos ou injetivos. Portanto, basta verificar que se $Y \in \operatorname{add}(M)$ é indecomponível, $(\mathrm{Y}, \mathrm{g})$ é sobrejetora.

Se $Y \in \operatorname{add}(M)$ é um indecomponível projetivo, não há nada que mostrar.

Para $Y$ indecomponível injetivo, será necessário mostrar antes que $(M, g)$ é sobrejetora.

Observe que, do Lema 3.6

$$
\left(D(\Lambda), P_{0}\right) \rightarrow(D(\Lambda), X)=0 \rightarrow 0 \text { é exata }
$$

pois $D(\Lambda)$ é injetivo, mas $X$ não é (se fosse, estaria em $\operatorname{add}(M))$.

E como $\Lambda$ é projetivo, $\left(\Lambda, P_{0}\right) \rightarrow(\Lambda, X) \rightarrow 0$ é exata.

Portanto,

$$
0 \rightarrow\left(D(\Lambda) \oplus \Lambda, P_{1}\right) \rightarrow\left(D(\Lambda) \oplus \Lambda, P_{0}\right) \rightarrow(D(\Lambda) \oplus \Lambda, X) \rightarrow 0 \text { é exata. }
$$

Com isso, para o módulo $D(\Lambda) \oplus \Lambda=M$, tem-se a exatidão da seqüência

$$
0 \rightarrow\left(M, P_{1}\right) \rightarrow\left(M, P_{0}\right) \rightarrow(M, X) \rightarrow 0
$$

Por fim considere então $\mathrm{Y}$ indecomponível injetivo. 
Para que

$$
\begin{aligned}
& (\mathrm{Y}, \mathrm{g}): \quad\left(\mathrm{Y}, \mathrm{P}_{\mathrm{O}}\right) \quad \rightarrow \quad(\mathrm{Y}, \mathrm{X}) \\
& \mathrm{h}: \mathrm{Y} \rightarrow \mathrm{P}_{0} \mapsto \mathrm{gh}: \mathrm{Y} \rightarrow \mathrm{X}
\end{aligned}
$$

seja sobrejetora, é necessário que, dado $\mathrm{t}: \mathrm{Y} \rightarrow \mathrm{X}$, exista $v: \mathrm{Y} \rightarrow \mathrm{P}_{\mathrm{O}}$ tal que $\mathrm{g} v=\mathrm{t}$.

Como $Y$ é injetivo, a seqüência $O \rightarrow Y \stackrel{i}{\hookrightarrow} M$ cinde, ou seja, existe um epimorfismo $j: M \rightarrow Y$ tal que $j i=I_{Y}$. Considere o diagrama

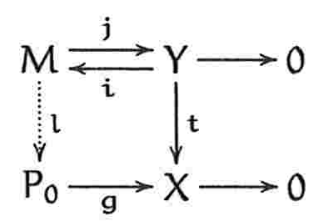

Recorde que $(M, g)$ é sobrejetora. Assim, como $t j \in(M, X)$, existe $l \in\left(M, P_{0}\right)$ tal que $\mathrm{gl}=\mathrm{t} j$. Então, dado $\mathrm{t} \in(\mathrm{Y}, \mathrm{X})$, existe $v=l i \in\left(\mathrm{Y}, \mathrm{P}_{0}\right)$ tal que

$$
g v=g(l i)=(g l) i=(t j) l=t(j i)=t_{Y}=t .
$$

Logo, $(\mathrm{Y}, \mathrm{g})$ é sobrejetora também no caso em que $\mathrm{Y}$ é injetivo indecomponível. $\mathrm{E}$ assim, para qualquer $Y \in \operatorname{add}(M)$, para qualquer $X$ indecomponível, é exata a seqüência

$$
0 \rightarrow\left(Y, P_{1}\right) \rightarrow\left(Y, P_{0}\right) \rightarrow(Y, X) \rightarrow 0
$$

de onde se conclui que se $X$ é indecomponível, há uma resolução de add(M)- aproximações com comprimento 2.

Se $X$ não é indecomponível, é soma direta de indecomponíveis, e as resoluções de $\operatorname{add}(M)$-aproximações de seus somandos induzem uma resolução de comprimento 2 para $\mathrm{X}$, conçluindo a demonstração.

\subsection{2 Álgebras coladas e Álgebras Laura.}

As colagens de álgebras foram introduzidas por Assem e Coelho([AC94]) para, segundo os autores, "relacionar a teoria de partições pré-projetivas e pré-injetivas introduzidas por Auslander e Smalø, com a teoria das álgebras inclinadas ('tilted'), introduzidas por Happel e Ringel". Posteriormente, outras propriedades das álgebras coladas foram estudadas em [AC03], onde também, ou principalmente, são apresentadas as álgebras laura (as definições são dadas mais a frente). 
Além da classe de álgebras coladas à direita e coladas à esquerda, a classe de álgebras laura generaliza outras importantes classes, como por exemplo, das álgebras fracamente shod (que por sua vez, contém a classe das álgebras quasi-tilted, e portanto as álgebras tilted).

O principal resultado desta seção é mais um exemplo dado em [CP04] do uso da técnica comentada naquele artigo, para o cálculo da dimensão de representação. Em [APT], os autores calculam, usando o mesmo método, a dimensão de representação para álgebras tilted e para álgebras estritamente laura (recorde que vale ainda a hipótese de que as álgebras são de tipo de representação infinito). Para estas e para as álgebras coladas vale que rep. $\operatorname{dim}(\Lambda)=3$. Neste último artigo ainda se mostra que se $\Lambda$ é quasi-tilted, então rep. $\operatorname{dim}(\Lambda) \leqslant 4$.

A definição original de álgebras coladas envolve conceitos que não convém ao escopo desta dissertação, portanto será assumido como definição a caracterização dada abaixo. Sua prova, bem como a definição original de álgebras coladas podem ser encontradas em [AC94, AC03]. Antes de enunciá-la, alguimas definições são necessárias.

Sejam $\mathrm{X}$ e $\mathrm{Y}$ dois $\Lambda$-módulos indecomponíveis. Um caminho de $\mathrm{X}$ a $\mathrm{Y}$ é uma seqüência (não necessariamente exata) de morfismos não-nulos

$$
X=X_{0} \rightarrow X_{1} \rightarrow X_{2} \rightarrow \ldots \rightarrow X_{n-1} \rightarrow X_{n}=Y, \text { onde } X_{i} \in \operatorname{ind}(\Lambda)
$$

Se existe um caminho de $X$ a $Y$, diz-se que $X$ é um predecessor de $Y$, e que $Y$ é um sucessor de $\mathrm{X}$. Esta situação é denotada por $\mathrm{X} \rightsquigarrow \mathrm{Y}$.

Observe que $X$ é sucessor e predecessor de si mesmo.

Para uma álgebra de Artin $\Lambda$, considere as subcategorias plenas de ind $(\Lambda)$ :

$\mathcal{L}_{\Lambda}=\{X \in \operatorname{ind}(\Lambda):$ se $Y$ é predecessor de $X$, então $\operatorname{pd}(Y) \leqslant 1\}$

$\mathcal{R}_{\Lambda}=\{X \in \operatorname{ind}(\Lambda):$ se $Y$ é sucessor de $X$, então id( $\left.Y) \leqslant 1\right\}$

Recorde que uma subcategoria $\mathcal{C}^{\prime}$ de $\mathcal{C}$ é cofinita em $\mathcal{C}$ quando só existe um múmero finito de objetos em $\mathcal{C} \backslash \mathfrak{C}^{\prime}$

Diz-se que uma proposição $\mathrm{P}$ sobre objetos de uma categoria $\mathrm{C}$ vale quase sempre em $\mathrm{C}$ quando $\mathrm{P}$ não é verdadeira somente para um subconjunto finito de objetos de $\mathcal{C}$. Em outras palavras, $\mathrm{P}$ vale quase sempre em $\mathcal{C}$ quando a subcategoria $\{X \in \mathcal{C}: P$ é verdadeira para $X\}$ é cofinita em $\mathcal{C}$. 
3.8 Proposição. Seja $\wedge$ uma álgebra de Artin.

1. $\mathcal{L}_{\Lambda}$ é fechada para predecessores e $\mathcal{R}_{\Lambda}$ é fechada para sucessores.

2. Sĩo equivalentes

(a) ^ é álgebra de Artin colada à esquerda;

(b) $\mathcal{L}_{\Lambda}$ é cofinita em ind $(\Lambda)$;

(c) $\operatorname{pd}(X) \leqslant 1$ quase sempre em $\operatorname{ind}(\Lambda)$.

3. São equivalentes

(a) ^ é álgebra de Artin colada à direita;

(b) $\mathcal{R}_{\Lambda}$ é cofinita em ind $(\Lambda)$;

(c) $\operatorname{id}(X) \leqslant 1$ quase sempre em $\operatorname{ind}(\Lambda)$.

Demonstração. Para 2 e 3, veja [AC94] e [AC03]. Para ver que $\mathcal{L}_{\Lambda}$ é fechada para predecessores, é necessário mostrar que para cada $X \in \mathcal{L}_{\Lambda}, \operatorname{pd}(T) \in \mathcal{L}_{\Lambda}$ para qualquer predecessor $T$ de $X$. Isso é equivalente a exigir que, para cada predecessor $Y$ de $T$, valha $\operatorname{pd}(Y) \leqslant 1$. Mas se $Y \rightsquigarrow T$, como $T \rightsquigarrow X$, então $Y \rightsquigarrow X$, e portanto $\operatorname{pd}(Y) \leqslant 1$, já que $X \in \mathcal{L}_{\Lambda}$. A outra afirmação é dual e tem demonstração semelhante.

3.9 Exemplo. Todas as álgebras de tipo finito são coladas. Mas a recíproca não é verdadeira. Este exemplo pode ser visto com detalhes em [AC94]. A álgebra de caminhos dada pelo quiver abaixo

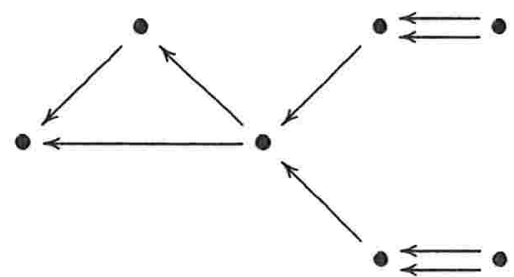

é uma colagem de duas álgebra de Kronecker com uma álgebra de radical quadrado nulo. Mas não é uma álgebra de tipo finito.

A demonstração do Lema abaixo pode ser encontrada em [AC03], e envolve os conceitos de caminhos de irredutíveis, e morfismos quase cindidos à esquerda e à direita. 
3.10 Lema. [AC03, 1.5] Seja $\wedge$ uma álgebra colada à esquerda. Se I é um injetivo indecomponivel, então existe um número finito de módulos $\mathrm{N} \in \mathcal{L}_{\Lambda}$ de modo que exista um caminho I $\rightsquigarrow$ N. Em outras palavras, para cada injetivo indecomponivel I, existe um número finito de sucessores de I em $\mathcal{L}_{\Lambda}$. Dualmente, para cada $\mathrm{P}$ projetivo indecomponível, existe um número finito de predecessores de $\mathrm{P}$ em $\mathcal{R}_{\Lambda}$.

Observação 3.11. O conjunto $\mathcal{X}_{\mathrm{I}}=\left\{\mathrm{N} \in \mathcal{L}_{\Lambda}: \mathrm{I} \rightsquigarrow \mathrm{N}\right\}$ é finito, para cada I injetivo indecomponível.

Portanto, se $\mathbf{X}$ denota o conjunto de todos os indecomponíveis injetivos em ind $(\Lambda)$, então $\mathcal{X}_{2}=\bigcup_{\mathrm{I} \in \mathbf{X}} \mathcal{X}_{\mathrm{I}}$ é um conjunto finito.

Observe que $\mathcal{X}_{2}$ é fechado para sucessores. Com efeito, se $\mathrm{N} \in \mathcal{X}_{2}$, então existe $\mathrm{I} \in \mathbf{X}$ tal que I $\rightsquigarrow N$. Se $N^{\prime}$ é sucessor de N, então, I $\rightsquigarrow N \rightsquigarrow N^{\prime}$, donde I $\rightsquigarrow N^{\prime}$.

Observe ainda que se I é injetivo indecomponível que está em $\mathcal{L}_{\Lambda}$, então $\mathrm{I} \in \mathcal{X}_{2}$, pois I é sucessor de si mesmo.

Com estas informações em mãos, é possível agora enunciar o resultado que calcula a dimensão de representação para álgebras coladas.

3.12 Teorema. Se $\wedge$ é uma álgebra de Artin de tipo infinito, colada à esquerda ou colada à direita, então rep. $\operatorname{dim}(\Lambda)=3$

Demonstração. Seja $\Lambda$ uma álgebra colada à esquerda. De acordo com o Teorema 2.21, é suficiente encontrar um gerador-cogerador de $\bmod (\Lambda)$ que é 2-aproximador.

Como $\mathcal{L}_{\Lambda}$ é cofinita em ind $(\Lambda)$, é finito o conjunto $\mathcal{X}_{1}=\operatorname{ind}(\Lambda) \backslash \mathcal{L}_{\Lambda}$.

Considere o conjunto finito

$$
\mathcal{X}=\mathcal{X}_{1} \cup \mathcal{X}_{2} \cup\{\mathrm{P}: \mathrm{P} \text { é projetivo indecomponível }\}
$$

onde $\mathcal{X}_{2}$ é o conjunto definido na Observação 3.11. Então $M=\bigoplus_{X \in \mathcal{X}}$ X é um geradorcogerador de $\bmod (\Lambda)$. Com efeito, todos os projetivos indecomponíveis são somandos de $M$, por construção, e se I é um indecomponível injetivo que não esteja em $\mathcal{L}_{\wedge}$ (estando portanto em $\mathcal{X}_{1}$ ), então estará em $\mathcal{X}_{2}$, conforme a observação acima.

Basta então mostrar que $M$ é um 2-aproximador, ou seja, existe uma resolução de $\operatorname{add}(M)$-aproximações com comprimento 2, para cada indecomponível $X$. 
Se $X \in \operatorname{add}(M)$, não há nada que provar. Seja, portanto, $X \notin \operatorname{add}(M)$. Então $X \in \mathcal{L}_{\Lambda}$, pois se não estivesse, deveria ser elemento de $\mathcal{X}_{1}$, e portanto, estaria em $\operatorname{add}(M)$.

Como $X \in \mathcal{L}_{\wedge}$ e não é projetivo, vale $\operatorname{pd}(X)=1$.

Seja $0 \rightarrow P_{1} \rightarrow P_{0} \rightarrow X \rightarrow 0$ uma resolução projetiva minimal de $X$.

Para que esta seqüência exata seja resolução de $\operatorname{add}(M)$-aproximações, deve ser exata a seqüência

$$
0 \rightarrow\left(\mathrm{Y}, \mathrm{P}_{1}\right) \rightarrow\left(\mathrm{Y}, \mathrm{P}_{0}\right) \rightarrow(\mathrm{Y}, \mathrm{X}) \rightarrow 0
$$

para cada $Y$ indecomponível de $\operatorname{add}(M)$.

Recordando a demonstração do Teorema 3.7, basta verificar que é exata a seqüência $\left(\mathrm{Y}, \mathrm{P}_{\mathrm{O}}\right) \rightarrow(\mathrm{Y}, \mathrm{X}) \rightarrow 0$

Isso é obviamente verdade se $Y$ é projetivo. Mas se $Y \in \mathcal{X}_{1}$ ou se $Y \in \mathcal{X}_{2}$, tem-se $(\mathrm{Y}, \mathrm{X})=0$. Com efeito, se $\mathrm{Y} \in \mathcal{X}_{1}$ e $\mathrm{f}: \mathrm{Y} \rightarrow \mathrm{X}$ é morfismo não nulo, $\mathrm{Y} \leadsto \mathrm{X}$. Mas $\mathrm{X} \in \mathcal{L}_{\Lambda}$, que é fechada para predecessores (Proposição 3.8). Neste caso, $Y \in \mathcal{L}_{\Lambda}$, contradição. Se, por outro lado, $\mathrm{Y} \in \mathcal{X}_{2}$ e $\mathrm{f}: \mathrm{Y} \rightarrow \mathrm{X}$ é morfismo não nulo, $\mathrm{Y} \rightsquigarrow \mathrm{X}$. Mas $\mathcal{X}_{2}$ é fechado para sucessores (Observação 3.11). Logo $X$ está em $\mathcal{X}_{2}$ e portanto, em add(M), outra contradição.

Portanto, fica estabelecido que se $\Lambda$ é álgebra colada à esquerda, rep. $\operatorname{dim}(\Lambda)=3$. Para uma álgebra colada à direita $\Lambda$, basta repetir os argumentos usando conceitos duais de resolução de $\operatorname{add}(M)$-aproximações e provas duais dos resultados usados nesta demonstração.

3.13 Definição. Uma álgebra de Artin $\Lambda$ é um Álgebra Laura quando a subcategoria plena $\mathcal{L}_{\Lambda} \cup \mathcal{R}_{\Lambda}$ é cofinita em ind $(\Lambda)$. Uma álgebra Laura é chamada Laura estrita quando não é quasi-tilted.

Os resultados a seguir estão demonstrados em [APT], e usam o Teorema 2.21 para construir o gerador-cogerador adequado.

\subsection{Proposição.}

1. Se $\Lambda$ é álgebra tilted ou laura estrita, então $\operatorname{rep} \cdot \operatorname{dim}(\Lambda)=3$;

2. Se $\Lambda$ é álgebra quasi-tilted, então rep. $\operatorname{dim}(\Lambda) \leqslant 4$. 


\subsection{Outros resultados}

Vários autores debruçaram-se sobre a dimensão de representação, e muitos resultados foram èncontrados. Este capítulo tem o objetivo de apresentar alguns destes resultados, sem a pretensão de esgotar o tema. A maior parte dos resultados será apenas enunciada, explicada a relevância do resultado e será indicado ao leitor onde mais informações podem ser encontradas. As álgebras em questão nesta seção continuam sendo álgebras de Artin de dimensão infinita não-semi-simples.

Sobre a finitude da dimensão de representação. Recorde que a dimensão de representação de uma álgebra $\wedge$ foi caracterizada como sendo o ínfimo de um conjunto, a saber, o conjunto das dimensões globais de todas as álgebras de endomorfismo de geradores-cogeradores de $\bmod (\Lambda)$ (veja o Corolário 2.16). Em princípio, aquele conjunto não precisaria ter um ínfimo, ou seja, para cada gerador-cogerador $M \in \bmod (\Lambda)$, poderia ocorrer que a dimensão global de $\operatorname{End}_{\wedge}(M)^{\text {op }}$ fosse infinita. Entretanto isso não ocorre.

Xi já especulava em [Xi02, 6.1] o uso de álgebras quase-hereditárias para demonstrar que álgebras sobre corpos têm dimensão de representação finita.

Com técnicas semelhantes, Osamu Iyama, em [Iya03], usando o fato de que a dimensão global de uma álgebra quase-hereditária é sempre finita, conseguiu mostrar que cada $\Lambda$ módulo $X$ finitamente gerado é somando de um módulo $M$ de modo que $\operatorname{End}_{\Lambda}(M)^{\text {op }}$ é quase-hereditária. Com isso, foi possível estabelecer o Teorema abaixo, que garante a finitude da dimensão de representação. Vamos especificar melhor esta observação. Informações relevantes sobre álgebras quase-hereditárias podem ser encontradas no artigo [DR89], de V. Dlab e C. M. Ringel, que fizeram uso deste conceito (introduzido por Cline-Parchall-Scott [CPS88a, CPS88b]) para o estudo das álgebras de Artin.

3.15 Definição. Um ideal J de um anel R é dito ideal hereditário quando:

1. $\mathrm{J}^{2}=\mathrm{J}$

2. $\mathrm{J} \operatorname{rad}(\mathrm{R}) \mathrm{J}=0$; 
3. ${ }_{\mathrm{R}} J$ é um projetivo

Uma álgebra de Artin $\Lambda$ é dita álgebra quase-hereditária quando existe uma cadeia de ideais

$$
\mathrm{J}_{0}=0 \subseteq \mathrm{J}_{1} \subseteq \mathrm{J}_{1} \subseteq \mathrm{J}_{2} \subseteq \ldots \subseteq \mathrm{J}_{\mathrm{n}}=\wedge
$$

tal que $\frac{\mathrm{J}_{i}}{\mathrm{~J}_{\mathrm{i}-1}}$ é ideal hereditário de $\frac{\Lambda}{\mathrm{J}_{\mathrm{i}-1}}$.

Exemplos de álgebras quase-hereditárias são as álgebras com dimensão global no máximo 2 ([DR89]).

A proposição abaixo foi demonstrada no artigo citado acima, e a prova consiste em encontrar uma certa cadeia de subcategorias de $\bmod (\Lambda)$, que fornecerão uma cadeia de ideais para a álgebra definida na proposição.

3.16 Proposição. [Iya03, 2.2] Seja X um ^-módulo finitamente gerado qualquer.

Defina $X_{0}=X$ e $X_{n+1}=\operatorname{rad}\left(\operatorname{End}_{\Lambda}\left(X_{n}\right)\right) X_{n} \subsetneq X_{n}$.

Seja $\mathrm{m}$ o menor inteiro tal que $\mathrm{X}_{\mathrm{m}}=0$.

Então, End ${ }_{\wedge}\left(\mathrm{X}_{0} \oplus \bigoplus_{\mathrm{i}=1}^{\mathrm{m}-1} \mathrm{X}_{\mathrm{i}}\right)^{\mathrm{op}}$ é uma álgebra quasi-hereditária.

3.17 Teorema. [Iya03, 1.2] Seja $\wedge$ uma álgebra de Artin. A dimensão de representação de $\wedge$ é finita.

Demonstração. Sejam $X$ e $Y$ como na Observação 2.9. Então $X_{0}=X \oplus Y$ é geradorcogerador de $\bmod (\Lambda)$, assim como $M=X_{0} \oplus N$, para cada $\Lambda$-módulo finitamente gerado N. Então, aplicando o teorema acima ao módulo $X_{0}$, este será somando direto de um $\Lambda$-módulo $M$ tal que $\operatorname{End}_{\Lambda}(M)^{\text {op }}$ é quasi-hereditária. Isto significa que existe pelo menos um gerador-cogerador $M=X_{0} \oplus N$ de $\bmod (\Lambda)$ de modo que $\operatorname{End}_{\Lambda}(M)^{\text {op }}$ tenha dimensão global finita.

Sobre equivalências, extensões e limitações Ainda em [Xi02], Xi buscava saber quando álgebras de certa forma equivalentes se relacionavam com relação à dimensão de representação. Ele havia definido o conceito de álgebras estavelmente hereditárias, que são álgebras cujos projetivos indecomponíveis têm seus submódulos indecomponíveis ou 
projetivos ou simples, e cujos injetivos indecomponíveis têm seus fatores indecomponíveis ou injetivos ou simples (Definição 3.4 daquele artigo). Estas álgebras generalizam as álgebras que são estavelmente equivalentes a álgebras hereditárias, e Xi mostrou que para álgebras estavelmente hereditárias, a dimensão de representação é no máximo 3.

Entretanto, ele queria mostrar que álgebras estavelmente equivalentes tinham mesma dimensão de representação. Em seu artigo ele conseguiu verificar que a dimensão de representação é invariante mediante equivalência estável de tipo de Morita, um caso particular de equivalência estável. Em dois artigos, de maneira indepentende, X. Guo ([Guo05]) e por A. Dugas ([Dug02], trabalhando com álgebras sem nós) provaram finalmente que a dimensão de representação é invariante segundo equivalência estável.

Quando se trata de álgebras derivadamente equivalentes, $\mathrm{Xi}$, mostra um exemplo em que a dimensão de representação não é invariante sob este tipo de equivalência ([Xi02, Observação 2 da seção 4]). Entretanto, a dimensão de representação de extensões triviais é preservada quando se tomam para álgebras derivadamente equivalentes ([Xi02, 4.2]).

F. U. Coelho e M. I. Platzeck aproveitam-se deste fato e do Teorema em [CP04, 3] para mostrar que se $\Lambda$ é uma álgebra tilted iterada, então rep. $\operatorname{dim}(T(\Lambda)) \leqslant 3$, onde $T(\Lambda)$ é a extensão trivial de $\Lambda$. Este Teorema citado diz que extensões triviais de álgebras hereditárias têm dimensão de representação no máximo 3. Estes autores também mostram que, sob certas condições, álgebras de Artin têm dimensão de representação limitada pela dimensão de representação de suas extensões por um ponto.

Um mergulho de radicais $f: \Lambda \rightarrow \Gamma$ da álgebra de Artin $\Lambda$ na álgebra de Artin $\Gamma$ é um monomorfismo que aplica o radical de Jacobson de $\Lambda$ sobrejetivamente no radical de $\Gamma$. Esta definição é necessária para o principal teorema em [EHIS04], que garante que se f é um mergulho de radicais como acima, e se $\Gamma$ é de tipo finito, então $\operatorname{rep} \operatorname{dim}(\Lambda) \leqslant 3$. Com este resultado, os autores provam que uma álgebra bisserial especial tem dimensão de representação no máximo 3 .

Um esforço grande em mostrar que a dimensão de representação separa os tipos de representação foi feito por Thorsten e outros, numa série de artigos, em que se calculam a dimensão de representação de várias álgebras ([BHS04, Hol03, Hol04]), conseguindo verificar que algumas álgebras mansas têm dimensão de representação no máximo 3. 
Outra limitação foi obtida por Auslander, quando mostrou que uma álgebra autoinjetiva $\Lambda$ com índice de nilpotência igual a $n$ satisfaz rep. $\operatorname{dim}(\Lambda) \leqslant n+1$. Como exemplo, Auslander considerou o anel de grupos k[G], onde $G$ é um grupo finito e $k$ é um corpo. Nesse sentido, Thorten, recentemente [HH05] encontrou que algumas álgebras de grupo $\left(C_{2} \times C_{2^{m}}\right)$ que são de tipo de representação selvagem (para $m \geqslant 3$ ) têm dimensão de representação no máximo 3 .

Sobre valores maiores para a dimensão de representação Como se pode perceber, havia muita expectativa de que a dimensão de representação fosse no máximo 3 , para toda álgebra de Artin. Muito tempo se pensou que isso fosse verdade, mas recentemente, Raphaël Rouquier apresentou dois artigos em que apresentava um exemplo de álgebra de Artin com dimensão de representação maior que 3. Os artigos [Rou03], [Rou05], do próprio Rouquier, e [KK05], de Henning Krause e de Dirk Kussin, tratam desse assunto.

Concluindo Muito ainda há o que desvendar a respeito da dimensão de representação. Aparentemente, esse número ainda não revelou toda informação que contém, muito menos há um meio simples de calculá-lo. Algumas pistas para desvendar mais sobre a dimensão de representação seriam conhecer os indecomponíveis do complemento de $\Lambda \oplus D(\Lambda)$ na composição do gerador-cogerador que dá o mínimo para a dimensão global de $\operatorname{End}{ }_{\wedge}(M)^{o p}$, observando sua distribuição no quíver de Auslander-Reiten; encontrar uma demonstração da finitude da dimensão de representação usando o Teorema 2.21, mostrando que sempre existe um gerador-cogerador r-aproximador; e todas as perguntas que Auslander mesmo elabora na última página do texto que deu início a essa dissertação. 


\section{Referências Bibliográficas}

[AC03] I. Assem and F.U. Coelho, Two-sided gluings of tilted algebras, J. Algebra (2003), no. 269, 156-479.

[AC94] — Glueing of tilted algebras, J. Pure Appl. Algebra 3 (1994), no. 93, $255-243$.

[AF92] F. W. Anderson and K. R. Fuller, Rings and categories of modules, 2 ed., Graduate Texts in Mathematics, no. 13, New York Springer-Verlag, Nova Iorque, 1992.

[APT] I. Assem, M. I. Platzeck, and S. Trepode, On the representation dimension of tilted and laura algebras.

[ARS95] M. Auslander, I. Reiten, and S. Smalø, Representation theory of artin algebras, Cambridge Stud. Adv. Math., no. 36, Cambridge Univ. Press, Cambridge, 1995.

[Ass97] I. Assem, Algèbres et modules, cours et exercices., Enseignement des Mathématiques, Les Presses de l'Université d'Ottawa, Ottawa, 1997.

[Aus66] M. Auslander, Coherent functors, Proc. Conf. Categorical Algebra (La Jolla, 1965), Berlin Springer, 1966, pp. 189-231.

[Aus71] — Representation dimension of artin algebras, Queen Mary College Mathematical Notes, Queen Mary College, Londres, 1971.

[BHS04] R. Bocian, T. Holm, and A. Skowroński, The representation dimension of domestic weakly symmetric algebras, Cent. Eur. J. Math 2 (2004), no. 1, 67-75. 
[CLS82] C. Cibils, F. Larrión, and L. Salmerón, Métodos diagramáticos en teoría de representaciones representaciones, Monografías del Inst. de Matemática de la UNAM, no. 11, UNAM, México, 1982.

[Coe92] F. U. Coelho, Uma introdução à teoria de representações de álgebras., Outubro 1992.

[CP04] F. U. Coelho and M. I. Platzeck, On the representation dimension of some classes of algebras, J. Algebra (2004), no. 275, 615-628.

[CPS88a] E. Cline, B. Parshall, and L. Scott, Algebraic stratification in representation categories, J. Algebra 117 (1988), no. 2, 504 - 521.

[CPS88b] — Finite dimensional algebras and highest weigh categories, J. Reine Angew. Math. (1988), no. 391, 85 - 99.

[DK94] Y. Drozd and V. Kirichenko, Finite-dimensional algebras, Springer-Verlag, Ber$\lim , 1994$.

[DR89] V. Dlab and C. M. Ringel, Quasi-hereditary algebras, Illinois J. Math. (1989), no. $33,280-291$.

[Dug02] A. S. Dugas, Representation dimension as a relative homological invariant of stable equivalence, 2002.

[EHIS04] K. Erdmann, T. Holm, O. Iyama, and J. Schröer, Radical embeddings and representation dimension, Adv. Math. (2004), no. 185, 159-177.

[Guo05] XiangQian Guo, Representation dimension: an invariant under stable equivalence, Trans. Amer. Math. Soc. 357 (2005), no. 8, 3255-3263.

[HH05] T. Holm and W. Hu, On the representation dimension of rank 2 group algebras and related algebras, 2005.

[Hol03] T. Holm, Representation dimension of some tame blocks of finite groups, Algebra Colloquium (2003), no. 10, 275-284. 
[Hol04] - The representation dimension of schur algebras: The tame case, Quart. J. Math 55 (2004), 477-490.

[IT02] K. Igusa and G. Todorov, On the finitistic global dimension conjecture, 2002.

[Iya03] O. Iyama, Finitness of representation dimension, Proc. Amer. Math. Soc. (2003), no. 131, 1011-1014.

[Jac89] N. Jacobson, Basic algebra, 2 ed., vol. 2, W. H. Freeman and Company, Nova iorque, 1989.

[KK05] H. Krause and D. Kussin, Rouquier's theorem on representation dimension, 2005.

[Rot79] J. J. Rotman, An introduction to homological algebra, Pure and Applied Mathematics, Academic Press, Inc, Nova Iorque-São Francisco-Londres, 1979.

[Rou03] R. Rouquier, Dimensions of triangulated categories, 2003.

[Rou05] — Representation dimension of exterior algebras, 2005.

[Xi00] C. Xi, On the representation dimension of finite-dimensional algebras, J. Algebra (2000), no. 226, 332-346.

[Xi02] _ Representation dimension and quasi-hereditary algebras, Adv. in Math. 2 (2002), no. 168, 193-212. 


\section{Índice Remissivo}

C-aproximações, 32

álgebra, 4

de Artin, 4

de Auslander, 19

Laura, 47

hereditária, 40

Laura estrita, 47

oposta, 5

quase-hereditária, 49

anel oposto, 5

apresentação, 9

bilinear, 12

caminho entre indecomponíveis, 44

categoria

aditiva, 11

cobertura projetiva, 6

dimensão

projetiva, 7

de representação, 22

dominante, 18

global, 7

injetiva, 7

envolvente injetiva, 6 funtor

aditivo, 12

coerente, 14

ideal hereditário, 48

injetivo

objeto, 16

isoclasses, 4

módulo

cogerador, 23

gerador, 23

indecomponível, 3

Morita-equivalência, 10

objeto

injetivo, 16

projetivo, 16

predecessor, 44

projetivo

objeto, 16

r-aproximador, 32

resolução

de $\mathcal{C}$-aproximações, 32

injetiva, 7

projetiva, 6 
seqüência

exata, 6

exata em $\mathcal{F}_{M}, 16$

soma

direta, 11

subcategoria

cofinita, 44

gerada por um módulo, 6

sucessor, 44

tipo de representação finito, 4 